\title{
A filling-in problem and moderate degenerations of minimal algebraic varieties
}

\author{
Shigeharu Takayama
}

\begin{abstract}
We study a family of minimal algebraic varieties over an open variety and consider conditions which imply the existence of a compactification with a mildly singular limit fiber. We solve a filling-in problem posed by Wang.
\end{abstract}

\section{Introduction}

The topic discuss in this paper is the existence or non-existence of a "much better" good minimal model for a given fiber space $f: X \rightarrow Y$ whose general fiber has a good minimal model. A good minimal model $f^{\prime}: X^{\prime} \rightarrow Y$ of $X$ over $Y$ consists of a birational map $X \rightarrow X^{\prime}$ over $Y$ and a total space $X^{\prime}$ with only canonical singularities, with a relatively semi-ample canonical divisor $K_{X^{\prime}}$ and with some other properties; see [Fuj16a, $\S 3]$ or Definition 2.1. In particular, the total space $X^{\prime}$ and general fibers $X_{y}^{\prime}$ are nice varieties. The expression "much better" means, informally, a mildness of the singularities of a special fiber of $f^{\prime}$. Our main technical result is as follows.

Theorem 1.1. Let $f: X \rightarrow Y$ be a projective surjective morphism with connected fibers between normal quasi-projective varieties $X$ and $Y$. Suppose that $f: X \rightarrow Y$ is weakly semi-stable ([AK00, Definition 0.1] or Definition 2.2) and that there exists a good minimal model $f^{\prime}: X^{\prime} \rightarrow Y$ of $X$ over $Y$. Let $0 \in Y$ be a point, and let $X_{0}=\bigcup_{i \in I} F_{i}$ be the decomposition into irreducible components of the fiber of $f$ over 0 . Suppose that for every large and divisible integer $m>0$, the plurigenera equality $\sum_{i \in I} P_{m}\left(F_{i}\right)=P_{m}\left(X_{y}\right)$ holds for a general point $y \in Y$.

Then the fiber $X_{0}^{\prime}$ of $f^{\prime}$ over 0 is birational to an irreducible component, say $F_{1}$, of $X_{0}$ by the birational map $X \rightarrow X^{\prime}$, and other components $F_{i}(i \in I \backslash\{1\}$ if $|I| \geqslant 2)$ are uniruled and contained in the stable base locus of $K_{X}: F_{i} \subset \operatorname{SBs}\left(K_{X}\right)$. Moreover, $X_{0}^{\prime}$ is normal with canonical singularities at worst and $K_{X_{0}^{\prime}}$ is semi-ample, and $P_{m}\left(F_{1}\right)=P_{m}\left(X_{0}^{\prime}\right)=P_{m}\left(X_{y}\right)$ holds for every integer $m>0$ and a general point $y \in Y$.

Here for a proper variety $V$, the $m$-genus $P_{m}(V)$ is defined by that of any smooth birational model $\widetilde{V}$; that is, $P_{m}(V)=h^{0}\left(\widetilde{V}, \mathcal{O}_{\widetilde{V}}\left(m K_{\widetilde{V}}\right)\right)$. Moreover, if $V$ has canonical singularities at worst, $P_{m}(V)=h^{0}\left(V, \mathcal{O}_{V}\left(m K_{V}\right)\right)$ holds for any integer $m>0$ [Nak04, II, Lemma 2.11]. We note that by [Tak07, Theorem 1.2], the inequality $\sum_{i \in I} P_{m}\left(F_{i}\right) \leqslant P_{m}\left(X_{y}\right)$ holds for every integer $m>0$

Received 19 October 2016, accepted in final form 2 August 2017.

2010 Mathematics Subject Classification 14D06, 32J25.

Keywords: filling-in problem, degeneration, plurigenera, good minimal model, canonical singularity, quasi-Hodge metric.

This journal is (C) Foundation Compositio Mathematica 2019. This article is distributed with Open Access under the terms of the Creative Commons Attribution Non-Commercial License, which permits non-commercial reuse, distribution, and reproduction in any medium, provided that the original work is properly cited. For commercial re-use, please contact the Foundation Compositio Mathematica.

The author is supported by Grant-in-Aid for Scientific Research (B) 16H03929. 


\section{FILLING-IN PROBLEM AND DEGENERATIONS OF VARIETIES}

and a general point $y \in Y$. So the plurigenera equality condition is an extreme case. The property $F_{i} \subset \operatorname{SBs}\left(K_{X}\right)$ means that $F_{i}$ is contained in the zero locus of any $s \in H^{0}\left(X, \mathcal{O}_{X}\left(m K_{X}\right)\right)$ for any integer $m>0$. The proof depends on an analysis of relative good minimal models by Fujino [Fuj16a] and the lower semi-continuity of plurigenera under deformations [Tak07]. It would be interesting to compare with [Fuj11] and [Tak08], where the case of Kodaira dimension zero over a curve is discussed. Here is a simpler statement.

Corollary 1.2. Let $f: X \rightarrow Y$ be a smooth projective morphism with connected fibers between smooth quasi-projective varieties $X$ and $Y$. Suppose that there exists a good minimal model $f^{\prime}: X^{\prime} \rightarrow Y$ of $X$ over $Y$. Then $f^{\prime}$ is flat, and every fiber of $f^{\prime}$ is normal and has canonical singularities at worst. In particular, if one fiber $X_{y}$ is of general type, then the existence of a good minimal model $f^{\prime}: X^{\prime} \rightarrow Y$ of $X$ over $Y$ is known, and $K_{X_{y}^{\prime}}$ becomes semi-ample and big for every $y \in Y$.

Corollary 1.2 seems to be quite reasonable and is expected. We will then apply Theorem 1.1 to a filling-in problem posed by Wang [Wan03], which is a major motivation to establish Theorem 1.1. The problem asks whether for a given fiber space $f: X \rightarrow C$ over a pointed curve $C$ with $0 \in C$ whose nearby fibers $X_{t}(t \neq 0)$ are all smooth and good minimal, the special fiber $X_{0}$ has canonical singularities at worst after possibly a finite base change and a birational modification along the special fiber if a possibly degenerate Kähler metric $g_{m}$ on $C \backslash\{0\}$ is incomplete at $0 \in C$. The metric $g_{m}$ relates to the curvature of a Hermitian metric on $\left.f_{*}\left(\oplus_{i=1}^{m} \mathcal{O}_{X}\left(i K_{X / C}\right)\right)\right|_{C \backslash\{0\}}$. To state our next result more precisely, let us recall the following definition.

Definition 1.3. Let $f: X \rightarrow C$ be a projective surjective morphism with connected fibers from a normal quasi-projective variety $X$ to a smooth affine curve $C$ with a point $0 \in C$. We set $C^{o}=C \backslash\{0\}$ and $X^{o}=f^{-1}\left(C^{o}\right)$ and suppose that $X^{o}$ is smooth and $f: X^{o} \rightarrow C^{o}$ is smooth. We let $X_{t}=f^{*} t$ be the scheme-theoretic fiber of $t \in C$ and suppose $\operatorname{dim} X_{t}=n$.

(1) The vector bundle $\left.\left(f_{*} \mathcal{O}_{X}\left(K_{X / C}\right)\right)\right|_{C^{o}}$ (to be more precise, it should be $f_{*} \mathcal{O}_{X^{o}}\left(K_{X^{o} / C^{o}}\right)$ ) admits a natural fiberwise $L^{2}$-metric, say $h$, which is given by

$$
h_{t}\left(u_{t}, v_{t}\right)=\int_{X_{t}}(-1)^{n^{2} / 2} u_{t} \wedge \overline{v_{t}}
$$

for $t \in C^{o}$ and $u_{t}, v_{t} \in H^{0}\left(X_{t}, \mathcal{O}_{X_{t}}\left(K_{X_{t}}\right)\right)=\left(f_{*} \mathcal{O}_{X}\left(K_{X / C}\right)\right)_{t}$. By Griffiths [Gri70], the curvature of $h$ is semi-positive in the sense of Griffiths. In particular, the curvature of $\operatorname{det} h$ on $\left.\operatorname{det}\left(f_{*} \mathcal{O}_{X}\left(K_{X / C}\right)\right)\right|_{C^{o}}$,

$$
\omega_{1}=\frac{\sqrt{-1}}{2 \pi} \bar{\partial} \partial \log \operatorname{det} h,
$$

is a semi-positive $(1,1)$-form on $C^{o}$ and can be regarded as a possibly degenerate Kähler metric on $C^{o}$. The pseudo-metric $\omega_{1}$ is called the quasi-Hodge metric on $C^{o}$ in [Wan03] and denoted by $g_{H}$ or $g_{1}$. We prefer the normalizing constant $\sqrt{-1} / 2 \pi$; cf. [Wan03, beginning of Section 2 , p. 59].

(2) [Wan03, Definition 3.1]. We suppose that $K_{X}$ is $\mathbb{Q}$-Cartier and suppose that there exists a divisor $D \in\left|m K_{X / C}\right|$ which is smooth over $C^{o}$ for some divisible integer $m>0$. Let $\pi: Y \rightarrow X$ be the (normalized) cyclic cover obtained by taking the $m$ th root of $D$; see [EV92, $\S 3$ ] or Item 3.8 below. Let $f_{Y}=f \circ \pi: Y \rightarrow C$ be the induced morphism, and let $Y^{o}=f_{Y}^{-1}\left(C^{o}\right)$. As we will see, $\pi_{*} \mathcal{O}_{Y^{o}}\left(K_{Y^{o}}\right)=\oplus_{i=1}^{m} \mathcal{O}_{X^{o}}\left(i K_{X^{o}}\right)$, and hence $f_{Y_{*}} \mathcal{O}_{Y^{o}}\left(K_{Y^{o}} / C^{o}\right)=f_{*}\left(\oplus_{i=1}^{m} \mathcal{O}_{X^{o}}\left(i K_{X^{o}} / C^{o}\right)\right)$ is non-zero. We obtain the pseudo-metric $\omega_{1}$ on $C^{o}$ for $f_{Y}: Y \rightarrow C$, and then define the $m$ th pseudo-metric $\omega_{m, D}$ for $f: X \rightarrow C$ associated with $D \in\left|m K_{X / C}\right|$ to be $\omega_{1}$ for $f_{Y}: Y \rightarrow C$. 


\section{S. TAKAYAMA}

The pseudo-metric $\omega_{m, D}$ is called the mth quasi-Hodge metric on $C^{o}$ in [Wan03, Definition 3.1], where it is denoted by $g_{m}$ (and " $D$ " is omitted).

Here are some additional explanations. We actually want to discuss quantities attached to $f: X^{o} \rightarrow C^{o}$, not to $f: X \rightarrow C$. The metric $h$ in Definition 1.3(1) is defined for the smooth proper morphism $f: X^{o} \rightarrow C^{o}$ without referring to its compactification $f: X \rightarrow C$. Using classical Hodge theory due to Schmid [Sch73], Wang [Wan03, Theorem 2.1, Remark 2.2] observed that the pseudo-metric $\omega_{1}$, and hence any $\omega_{m, D}$, either has Poincaré growth or is incomplete at $0 \in C$. As for Definition 1.3(2), this pluricanonical version of Definition 1.3(1) is introduced because the direct image $f_{*} \mathcal{O}_{X^{o}}\left(K_{X^{o}} C^{o}\right)$ may be zero, and because even if it is non-zero, the geometric genus of the general fibers (or a variation of Hodge structures) contains only little information in general. The existence of such $D \in\left|m K_{X / C}\right|$ in Definition 1.3(2) can be confirmed, for example in the case that $f: X \rightarrow C$ is relatively good minimal and $m$ is large and divisible, so that $m K_{X}$ is Cartier and $\left|m K_{X}\right|$ is base-point free, possibly after replacing $C$ by a smaller affine neighborhood of $0 \in C$. This is the case we will actually deal with.

With these definitions in mind, our main result can be stated as follows (see Theorem 4.1 for a more precise statement).

Theorem 1.4. Let $f: X \rightarrow C$ be as in Definition 1.3, and suppose that any smooth fiber $X_{t}$ $\left(t \in C^{o}\right)$ has semi-ample canonical bundle. Then the following three conditions are equivalent, after possibly a finite base change and a birational modification along the special fiber (for example, after taking a semi-stable reduction and passing to a good minimal model):

(1) The special fiber $X_{0}$ has canonical singularities at worst, and $K_{X_{0}}$ is semi-ample.

(2) The pseudo-metric $\omega_{m, D}$ on $C^{o}$ is incomplete at $0 \in C$ for every large and divisible integer $m$ and every general $D \in\left|m K_{X / C}\right|$.

(3) For every integer $m>0$, the plurigenera equality $\sum_{i \in I} P_{m}\left(F_{i}\right)=P_{m}\left(X_{t}\right)$ holds for any $t \in C^{o}$, where $X_{0}=\sum_{i \in I} F_{i}$ is the decomposition into irreducible components.

Wang [Wan03, Proposition 1.3] proved the implication $(1) \Rightarrow(2)$ of Theorem 1.4, namely that the existence of a geometrically nice model implies a differential-geometric property, and posed the converse as a problem. He also proposed an approach to attack the implication $(2) \Rightarrow(1)$, namely to consider the condition in (3) and use the minimal model program; see [Wan03, $\S 3$. Our strategy for the proof of Theorem 1.4 is as follows. We can easily deduce that conditions (1) and (3) are equivalent by virtue of Theorem 1.1. To obtain the implication $(2) \Rightarrow(1)$, we shall suppose to the contrary that conditions (3) and (1) do not hold (even after taking a semistable reduction and passing to a good minimal model). Then $H^{0}\left(X_{0},\left.\mathcal{O}_{X}\left(m K_{X}\right)\right|_{X_{0}}\right)$ is strictly bigger than $\oplus_{i \in I} H^{0}\left(F_{i}, \mathcal{O}_{F_{i}}\left(m K_{F_{i}}\right)\right)$, where $X_{0}=\sum_{i \in I} F_{i}$ is the decomposition into irreducible components. As is well known, the fiberwise $L^{2}$-metrics such as $h$ in Definition 1.3(1) have singularities arisen from not only the singularities of the fiber $X_{0}$, but also the difference between $H^{0}\left(X_{0},\left.\mathcal{O}_{X}\left(m K_{X}\right)\right|_{X_{0}}\right)$ and $\oplus_{i \in I} H^{0}\left(F_{i}, \mathcal{O}_{F_{i}}\left(m K_{F_{i}}\right)\right)$. We actually estimate a divergence of the fiberwise $L^{2}$-metric $h$ in Proposition 4.7. We can then deduce that the curvature forms, such as $\omega_{1}$ and $\omega_{m, D}$, are still singular at $0 \in C$ and in fact are complete at $0 \in C$, as we already know possible types of asymptotics of $\omega_{1}$ and $\omega_{m, D}$ at $0 \in C$ by [Wan03, Theorem 2.1, Remark 2.2], [Yos10].

The origin of Wang's problem comes from the studies of degenerations of elliptic curves, abelian varieties and K3 surfaces; these are all nowadays classical. In the case of degenerations 
of curves with genus $\geqslant 2$, he confirmed that a smooth filling-in is always possible [Wan03, Theorem 1.4] as an analogue of those three classically known cases. However, it is known (refer to [Wan03, Theorem 1.5]) that it is too much to expect that one can fill in a family with smooth $X_{0}$ in the general case in the situation of Theorem 1.4; that is, one needs to allow at least mild singularities on $X_{0}$. For an interesting special case when $K_{X_{t}}=\mathcal{O}_{X_{t}}$ for $t \in C^{o}$, that is, degenerations of Calabi-Yau type manifolds, this problem has recently been confirmed by Tosatti [Tos15], the author [Tak15] and Zhang [Zha16, §2]. This special case is particularly interesting because of further interactions with Kähler-Einstein geometry, Donaldson-Sun's theory on the GromovHausdorff convergence of a certain class of Kähler manifolds [DS14], and maybe more. We also expect these interactions in the case of the present paper. It would be an interesting future problem.

\section{Simultaneous minimal models}

In this section, we discuss the regularity of a special fiber of a relative good minimal model and prove Theorem 1.1. We first recall some basic definitions.

Definition 2.1 (Good minimal model, see [Fuj16a, $\S 3$ ] for more details). Let $f: X \rightarrow Y$ be a projective surjective morphism of normal quasi-projective varieties with connected fibers. Suppose that $X$ has canonical singularities at worst. A normal variety $X^{\prime}$, a morphism $f^{\prime}: X^{\prime} \rightarrow Y$ and a rational map $\varphi: X \rightarrow X^{\prime}$ over $Y$ are called a minimal model of $X$ over $Y$ if

(1) $X^{\prime}$ is $\mathbb{Q}$-factorial,

(2) $f^{\prime}$ is projective,

(3) $\varphi$ is birational and $\varphi^{-1}$ has no exceptional divisors,

(4) $K_{X^{\prime}}$ is $f^{\prime}$-nef, and

(5) $a(E, X)<a\left(E, X^{\prime}\right)$ for every $\varphi$-exceptional divisor $E \subset X$, where $a(E, X)$ is the discrepancy of $E$ over $X$ [KM98, Definition 2.22].

Furthermore, if $K_{X^{\prime}}$ is $f^{\prime}$-semi-ample, then $X^{\prime}$ is called a (relative) good minimal model of $X$ over $Y$.

In particular, minimal models $X^{\prime}$ have only canonical singularities. We refer to [Kol13] for singularities in the minimal model program, such as canonical singularities [Kol13, §2.1] (or $[\mathrm{KM} 98, \S 2.3])$ and semi-log-canonical singularities [Kol13, § 5.2].

Definition 2.2 (Weakly semi-stable, [Fuj16a, Theorem 4.4], [AK00, Definition 0.1]). The morphism $f: X \rightarrow Y$ as in Definition 2.1 is said to be weakly semi-stable if

(1) $Y$ is smooth,

(2) there exist toroidal embeddings without self-intersection $\left(U_{X} \subset X\right)$ and $\left(U_{Y} \subset Y\right)$ such that $U_{X}=f^{-1}\left(U_{Y}\right)$,

(3) all the fibers of $f$ are reduced, and

(4) $f:\left(U_{X} \subset X\right) \rightarrow\left(U_{Y} \subset Y\right)$ is toroidal and equi-dimensional.

A weakly semi-stable morphism $f: X \rightarrow Y$ is said to be semi-stable if $X$ is smooth.

We refer to [AK00] for more technical terms on toroidal geometry. If $f$ is weakly semi-stable, it follows that $X$ has rational Gorenstein singularities at worst [AK00, $\S 6]$ (in particular, it has canonical singularities at worst [KM98, Corollary 5.24]). 


\section{S. TAKAYAMA}

We next recall two main technical tools in the proof of Theorem 1.1. The first one deals with basic properties of the good minimal model $f^{\prime}: X^{\prime} \rightarrow Y$ starting from a weakly semi-stable $f: X \rightarrow Y$; it was obtained by Fujino [Fuj16a] (some key properties were already obtained by Abramovich-Karu $[\mathrm{AK} 00, \S 6])$.

Lemma 2.3. Let everything be as in Theorem 1.1.

(1) [Fuj16a, Theorem 4.4], see also [Fuj16b, Step 3, p. 262, lines 20-21]. The total space $X$ has only rational Gorenstein singularities. Let $y \in Y$ be an arbitrary point, and let $C$ be a smooth curve passing through $y$ such that $C=H_{1} \cap H_{2} \cap \cdots \cap H_{\operatorname{dim} Y-1}$, where every $H_{i}$ is a general smooth ample Cartier divisor on $Y$. Then $X_{C}=X \times_{Y} C \rightarrow C$ is also weakly semi-stable [Kar00, Lemma 2.12], and $X_{C}^{\prime}=X^{\prime} \times_{Y} C$ is normal and has only canonical singularities.

(2) [Fuj16a, Remark 4.3]. The morphism $f^{\prime}: X^{\prime} \rightarrow Y$ is equi-dimensional and flat. For every $y \in Y$, the scheme-theoretic fiber $X_{y}^{\prime}$ of $f^{\prime}$ has only semi-log-canonical singularities (which are at least reduced), and $\left.\mathcal{O}_{X^{\prime}}\left(m K_{X^{\prime}}\right)\right|_{X_{y}^{\prime}} \cong \mathcal{O}_{X_{y}^{\prime}}\left(m K_{X_{y}^{\prime}}\right)$ for every integer $m>0$. In particular $K_{X_{y}^{\prime}}$ is $\mathbb{Q}$-Cartier and semi-ample.

(3) [Fuj16a, §4, Proof of Theorem 1.6, Step 3]. For every integer $m>0$, the dimension $h^{0}\left(X_{y}^{\prime},\left.\mathcal{O}_{X^{\prime}}\left(m K_{X^{\prime}}\right)\right|_{X_{y}^{\prime}}\right)$ is independent of $y \in Y$. We also note that $h^{0}\left(X_{y}^{\prime}, \mathcal{O}_{X_{y}^{\prime}}\left(m K_{X_{y}^{\prime}}\right)\right)=$ $h^{0}\left(X_{y}, \mathcal{O}_{X_{y}}\left(m K_{X_{y}}\right)\right)=P_{m}\left(X_{y}\right)$ for general $y \in Y$.

Lemma 2.3(2) implies the flatness of good minimal models $f^{\prime}$ and the semi-ampleness of every $K_{X_{y}^{\prime}}$ in Theorem 1.1, Corollary 1.2 and Theorem 1.4.

The second technical tool is an extension of pluricanonical forms. Our proof of Theorem 1.1 will show that the invariance of plurigenera $P_{m}\left(X_{y}\right)$ of the fibers [Siu98, Siu02] (see also [Kaw99, Nak04, Pău07, Tak07]) controls the fibers of $f^{\prime}$. We will formulate it in a general form. We may denote the pluricanonical bundle $K_{X}^{\otimes m}$ by $m K_{X}$ by an abuse of notation.

Theorem 2.4 ([Tak07, Theorem 3.1]). Let $X$ be a complex manifold, $\Delta=\{|t|<1\}$ the unit disk in $\mathbb{C}$ (or an open Riemann surface) and $f: X \rightarrow \Delta$ a projective surjective morphism with connected fibers. Assume that the central fiber $X_{0}$ has its prime decomposition in a form of $X_{0}=\sum_{i \in I} F_{i}+\sum_{j \in J} k_{j} F_{j}^{\prime}$ such that $F:=\sum_{i \in I} F_{i}$ is a disjoint union of smooth divisors, where the $k_{j} \geqslant 1$ are integers. Let $m$ be a positive integer and $s \in H^{0}\left(F, m K_{F}\right)=\oplus_{i \in I} H^{0}\left(F_{i}, m K_{F_{i}}\right)$. Then $s \wedge(d t)^{m} \in H^{0}\left(F,\left.m K_{X}\right|_{F}\right)$ can be extended to an element of $H^{0}\left(X, m K_{X}\right)$. In particular, $\sum_{i \in I} P_{m}\left(F_{i}\right) \leqslant P_{m}\left(X_{t}\right)$ holds for a general fiber $X_{t}$.

Let us explain the wedge product $* \wedge(d t)^{m}$ in the statement. We regard the coordinate function $t$ on $\Delta$ as a holomorphic function on $X$ via $f: X \rightarrow \Delta$. We have a holomorphic 1-form $d t \in H^{0}\left(X, \Omega_{X}^{1}\right)$ with some zeros on $X_{0}$. On $F$, more precisely on every $F_{i}(i \in I)$, we have a sheaf homomorphism $\Phi:\left.K_{F} \rightarrow K_{X}\right|_{F}$ defined by taking the wedge product with $d t$ as follows. Let $n+1$ be the dimension of $X$. Let us take local coordinates $z_{1}, \ldots, z_{n}, w$ of $X$ such that $F=\{w=0\}$ and that $t=\widetilde{\zeta} w$ for a holomorphic function $\widetilde{\zeta}=\widetilde{\zeta}\left(z_{1}, \ldots, z_{n}, w\right)$. Then $d t=\widetilde{\zeta} d w+w d \widetilde{\zeta}$. In these local coordinates, the divisor $X_{0}-F=\sum_{j \in J} k_{j} F_{j}^{\prime}=: F^{\prime}$ on $X$ is defined by $\widetilde{\zeta}$, and the divisor $\left.\left(X_{0}-F\right)\right|_{F}=\left.F^{\prime}\right|_{F}$ on $F$ is defined by $\zeta=\zeta\left(z_{1}, \ldots, z_{n}\right):=\widetilde{\zeta}\left(z_{1}, \ldots, z_{n}, 0\right)$. For a local holomorphic $n$-form $s=h\left(z_{1}, \ldots, z_{n}\right) d z_{1} \wedge \cdots \wedge d z_{n}$ on $F$, we take an arbitrary extension $\widetilde{s}$ of $s$ as a local holomorphic $n$-form on $X$. We can write it as $\widetilde{s}=\widetilde{h}\left(z_{1}, \ldots, z_{n}, w\right) d z_{1} \wedge \cdots \wedge d z_{n}+(n$-forms including $d w)$ for a local holomorphic extension $\widetilde{h}$ of $h$. Then, by noting that $w=0$ on $F$, we 
can define $\Phi:\left.K_{F} \rightarrow K_{X}\right|_{F}$ by

$$
\Phi(s)=\left.(\widetilde{s} \wedge d t)\right|_{F}=\left.\zeta \cdot h \cdot\left(d z_{1} \wedge \cdots \wedge d z_{n} \wedge d w\right)\right|_{F} .
$$

We shall also write $\Phi(s)=s \wedge d t$ by an abuse of notation. We can see, by the local computation above, that $\Phi$ has a zero, namely has degeneracy along the divisor $\left.F^{\prime}\right|_{F}(=\{\zeta=0\}$ locally). The intrinsic form of $\Phi$ is as follows. As $X_{0}=F+F^{\prime} \sim 0$ (that is, the divisor is linearly equivalent to zero), the adjunction formula $K_{F}=\left.\left(K_{X}+F\right)\right|_{F}$ tells us $K_{F}=\left.K_{X}\right|_{F}-\left.F^{\prime}\right|_{F}$. Hence, there exists a natural homomorphism $\left.K_{F} \rightarrow K_{X}\right|_{F}$ given by a product with a section of $H^{0}\left(F, \mathcal{O}_{F}\left(\left.F^{\prime}\right|_{F}\right)\right)$ vanishing exactly along $\left.F^{\prime}\right|_{F}$. This $\Phi:\left.K_{F} \rightarrow K_{X}\right|_{F}$ induces other sheaf homomorphisms and linear maps of cohomology groups. For example, for every integer $m>0$, we have an injection $H^{0}\left(F, m K_{F}\right) \rightarrow H^{0}\left(F,\left.m K_{X}\right|_{F}\right)$ defined by $s \mapsto s \wedge(d t)^{m}$. If we take $s \in H^{0}\left(F, m K_{F}\right)$ and write $s=h\left(z_{1}, \ldots, z_{n}\right)\left(d z_{1} \wedge \cdots \wedge d z_{n}\right)^{\otimes m}$ locally on $F$, then it is (with the same symbol)

$$
\Phi(s)=\left.\left(\widetilde{s} \wedge(d t)^{m}\right)\right|_{F}=\left.\zeta^{m} \cdot h \cdot\left(d z_{1} \wedge \cdots \wedge d z_{n} \wedge d w\right)^{\otimes m}\right|_{F} .
$$

We are now ready to prove Theorem 1.1.

Proof of Theorem 1.1. We set $J=\left\{i \in I ; \kappa\left(F_{j}\right) \geqslant 0\right\}$. Here, for a proper variety $V$, the Kodaira dimension $\kappa(V)$ is defined to be that of any smooth birational model $\widetilde{V}$ of $V$. As $\kappa\left(X_{y}\right) \geqslant 0$ for a general $y$, we have $\sum_{i \in I} P_{m}\left(F_{i}\right)=P_{m}\left(X_{y}\right) \neq 0$ for some $m>0$. It also implies that $J$ is non-empty.

Step 1. We recall a uniruledness criterion, which is a particular case of [Kaw91, Theorem 1] mentioned in that theorem as a prototype. An irreducible component $F$ of a fiber $X_{y}$ of $f$ is uniruled if $F$ is contained in the exceptional locus of a divisorial contraction or in a flipping contraction by the $K_{X}$-minimal model program (MMP) over $Y$ to obtain $\varphi: X \rightarrow-X^{\prime}$. As every component $F_{j}(j \in J)$ is not uniruled, $F_{j}$ is not contracted by the process to obtain $\varphi: X \rightarrow X^{\prime}$. In particular, $\left.\varphi\right|_{F_{j}}$ gives a birational map of $F_{j}$ as $F_{j}$ is mapped birationally in each step. Let $F_{j}^{\prime} \subset X_{0}^{\prime}$ be the irreducible component birational to $F_{j}$ (notice that $f$ and $f^{\prime}$ have equidimensional and reduced fibers). If we can prove $X_{0}^{\prime}=\bigcup_{j \in J} F_{j}^{\prime}$, then other components $F_{i}$ $(i \in I \backslash J)$ are contracted by the process to obtain $\varphi: X \rightarrow X^{\prime}$, and then they are uniruled.

Step 2. Since our assertion is local over $Y$, we may suppose that $Y$ is affine and $K_{Y}$ is trivial. We take a general smooth affine curve $C$ passing through $0 \in Y$ as in Lemma 2.3(1). Let us denote the induced morphisms by $g: Z:=X \times_{Y} C \rightarrow C$ and $g^{\prime}: Z^{\prime}:=X^{\prime} \times_{Y} C \rightarrow C$. The special fiber $Z_{0}^{\prime}$ of $g^{\prime}$ over 0 is $Z_{0}^{\prime}=X_{0}^{\prime}$. We know $Z_{0}^{\prime} \supset \bigcup_{j \in J} F_{j}^{\prime}$, at least. We take a log-resolution $\pi: Z^{+} \rightarrow Z^{\prime}$ of the pair $\left(Z^{\prime}, Z_{0}^{\prime}\right)$ such that strict transforms of irreducible components of $Z_{0}^{\prime}$ are mutually disjoint. Let $g^{+}=g^{\prime} \circ \pi: Z^{+} \rightarrow C$ be the induced morphism:

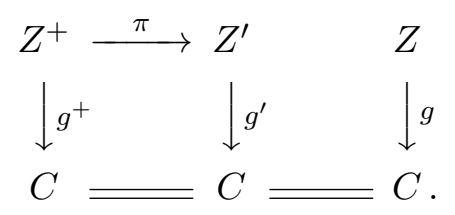

The birational map $\varphi: X \rightarrow X^{\prime}$ induces a birational map $Z \rightarrow Z^{\prime}$. We have the decomposition into irreducible components of the special fiber $Z_{0}^{+}=\pi^{*} Z_{0}^{\prime}=F^{+}+M+\sum_{k} c_{k} E_{k}$, where $F^{+}=\sum_{j \in J} F_{j}^{+}$and $F_{j}^{+}$is the strict transform of $F_{j}^{\prime}$, the component $M$ is the strict transform of $Z_{0}^{\prime}-\sum_{j \in J} F_{j}^{\prime}$ (with coefficient 1 if it is non-empty), the $E_{k}$ are $\pi$-exceptional prime divisors and the $c_{k}$ are non-negative integers. We will eventually show that $M=0$ in Step 3 and $|J|=1$ 


\section{S. TAKAYAMA}

in Step 4.

Step 3. We shall show that $Z_{0}^{\prime}\left(=X_{0}^{\prime}\right)=\bigcup_{j \in J} F_{j}^{\prime}$. We suppose to the contrary that there exists an irreducible component $M_{0}^{\prime} \subset Z_{0}^{\prime}$ other than the $F_{j}^{\prime}$, and let $M_{0}^{+}$be the strict transform of $M_{0}^{\prime}$.

As $K_{Z^{\prime}}=\left.K_{X^{\prime}}\right|_{Z^{\prime}}$ is $g^{\prime}$-semi-ample (and $Z^{\prime}$ has canonical singularities at worst, Lemma 2.3(1)), there exist a large and sufficiently divisible integer $m>0$ and a section $s \in H^{0}\left(Z^{+}, m K_{Z^{+}}\right)$such that $s \not \equiv 0$ on $M_{0}^{+}$. On the other hand, by Theorem 2.4, every element of $\left(\oplus_{j \in J} H^{0}\left(F_{j}^{+}, m K_{F_{j}^{+}}\right) \oplus\right.$ $H^{0}\left(M_{0}^{+}, m K_{M_{0}^{+}}\right)$can be extended to an element of $H^{0}\left(Z^{+}, m K_{Z^{+}}\right)$in an appropriate manner. In particular, every element of $\left(\oplus_{j \in J} H^{0}\left(F_{j}^{+}, m K_{F_{j}^{+}}\right)\right) \oplus\{0\}$ can be extended to an element of $H^{0}\left(Z^{+}, m K_{Z^{+}}\right)$. These sections are different from the one $s \in H^{0}\left(Z^{+}, m K_{Z^{+}}\right)$such that $s \not \equiv 0$ on $M_{0}^{+}$. We can also see that $\operatorname{Bs}\left|m K_{Z^{+}}\right| \subset \operatorname{Exc}(\pi)$ for any $m>0$.

Let $\alpha: H^{0}\left(Z^{+}, m K_{Z^{+}}\right) \otimes_{H^{0}\left(C, \mathcal{O}_{C}\right)} \mathcal{O}_{C} \rightarrow g_{*}^{+}\left(m K_{Z^{+}}\right) \cong g_{*}^{\prime}\left(m K_{Z^{\prime}}\right)$ be the naturally induced homomorphism. The sheaf $g_{*}^{\prime}\left(m K_{Z^{\prime}}\right)$ is locally free on $C$ and of rank $P_{m}\left(X_{y}\right)$, where $y \in C$ is an arbitrary general point. Our previous argument shows that the map $\alpha$ at $0 \in C$ (and hence at nearby $y \in C$ ) has rank at least $\sum_{j \in J} P_{m}\left(F_{j}^{+}\right)+1$. As $y \in C(\subset Y)$ is general, $P_{m}\left(Z_{y}^{+}\right)=P_{m}\left(X_{y}\right)$ holds since $Z_{y}^{+}$and $X_{y}$ are birational and have only canonical singularities. As $F_{j}$ is birational to $F_{j}^{\prime}$ and $F_{j}^{+}$, one has $P_{m}\left(F_{j}\right)=P_{m}\left(F_{j}^{+}\right)$and by assumption $\sum_{j \in J} P_{m}\left(F_{j}\right)=P_{m}\left(X_{y}\right)$ for $y \in C(\subset Y)$ general. This contradicts the inequality $P_{m}\left(X_{y}\right) \geqslant \sum_{j \in J} P_{m}\left(F_{j}^{+}\right)+1$.

Step 4. We shall show that $Z_{0}^{\prime}\left(=X_{0}^{\prime}\right)$ is normal, which in particular implies $|J|=1$. By Lemma 2.3(2), the fiber $Z_{0}^{\prime}$ is semi-log-canonical; in particular, it is demi-normal [Kol13, DefinitionLemma 5.10]. A demi-normal scheme is normal if and only if it is regular in codimension 1 [Kol13, Definition 5.1]. Hence, we focus on codimension 2 subvarieties of $Z^{\prime}$ contained in $Z_{0}^{\prime}$. A canonical singularity in a codimension 2 generic point is (i) smooth, or (ii) an ADE-type singularity, in view of Lemma 2.6(1) below. Suppose to the contrary that $Z_{0}^{\prime}$ is singular in codimension 1, and take an irreducible component $B$ of $\operatorname{Sing}\left(Z_{0}^{\prime}\right)$ with $\operatorname{dim} B=n-1$, where $n=\operatorname{dim} X-\operatorname{dim} Y$. As $Z^{\prime}$ has canonical singularities at worst, it can happen along the generic point of $B$ that either (i) $Z^{\prime}$ is smooth, or (ii) $Z^{\prime}$ is an ADE-type singularity, in view of Lemma 2.6(1). Let $U$ be an arbitrary open neighborhood of the generic point of $B$ in $Z^{\prime}$; then $U$ is a Zariski-open subset of $Z^{\prime}$ such that $\operatorname{dim}(B \backslash U)=\operatorname{dim} B-1$ and $Z^{\prime} \backslash U$ contains $\operatorname{Sing}\left(Z_{0}^{\prime}\right) \backslash B$. We are only interested in the generic point of $B$ and hence may shrink $U$ to a smaller Zariski-open subset having the same properties.

Let $m>0$ be a large and divisible integer such that $m K_{Z^{\prime}}$ is Cartier and base-point free. We treat the cases (i) and (ii) separately depending on the type of singularities of $Z^{\prime}$ along $B$.

(i) We choose the resolution $\pi: Z^{+} \rightarrow Z^{\prime}$ in Step 2 as follows. We first take a partial resolution $\pi_{1}: Z^{1} \rightarrow Z^{\prime}$ as the blow-up of $Z^{\prime}$ along $B$. We note that $Z_{0}^{\prime}$ has semi-log-canonical singularities at worst, and that a semi-log-canonical singularity in codimension 1 is an ordinary node. Hence, if we blow up $Z^{\prime}$ along $B$, then the strict transform of $Z_{0}^{\prime}$ is separated on the inverse image of $U$ (possibly shrinking $U$ a bit). We then take a further log-resolution $\pi_{2}: Z^{+} \rightarrow Z^{1}$ of the pair $\left(Z^{1}, \pi_{1}^{*} Z_{0}^{\prime}\right)$ such that $\pi_{2}$ is isomorphic over $\pi_{1}^{-1}(U)$. We take $\pi=\pi_{1} \circ \pi_{2}: Z^{+} \rightarrow Z^{\prime}$.

By adjunction, we have $K_{Z^{+}}=\pi^{*} K_{Z^{\prime}}+E_{1}$ over $U$, where $E_{1}$ is the unique $\pi$-exceptional prime divisor with $\pi\left(E_{1}\right)=B$. Then, by the base-point freeness of $m K_{Z^{\prime}}$, we can find a section $s \in H^{0}\left(Z^{+}, m K_{Z^{+}}\right)$such that the zero divisor of $\left.s\right|_{\pi^{-1}(U)}$ is exactly $m E_{1} \cap \pi^{-1}(U)$ (by shrink- 


\section{FiLling-IN PROBLEM AND DEGENERATIONS OF VARIETIES}

ing $U$ if necessary). On the other hand, we have $Z_{0}^{+}=F^{+}+2 E_{1}$ over $U$, where $F^{+}$is given at the end of Step 2 (note that the semi-log-canonical singularity in codimension 1 is an ordinary node, which gives the coefficient 2). We also see that $F^{+} \cap E_{1} \neq \emptyset$.

Then by Theorem 2.4 again, every element of $\oplus_{j \in J} H^{0}\left(F_{j}^{+}, m K_{F_{j}^{+}}\right)$can be extended to an element of $H^{0}\left(Z^{+}, m K_{Z^{+}}\right)$. Let $W \subset H^{0}\left(Z^{+}, m K_{Z^{+}}\right)$be the subspace generated by the sections obtained by the extensions from $\oplus_{j \in J} H^{0}\left(F_{j}^{+}, m K_{F_{j}^{+}}\right)$. On the other hand, if an irreducible component $F_{1}^{+}$of $F^{+}$intersects with $E_{1}$, we can see that every element of $W$ vanishes at least $2 m$ times along $F_{1}^{+} \cap E_{1}$ or vanishes identically on $F_{1}^{+}$. In fact, by the construction of the extension in Theorem 2.4, every element in $W$ vanishes along $\left\{\zeta^{m}=0\right\}$ at least, and $\{\zeta=0\}=2\left(F_{1}^{+} \cap E_{1}\right)$, which itself is a consequence of the decomposition $Z_{0}^{+}=F^{+}+2 E_{1}$. Thus $s \notin W$. Then by the dimension-counting argument as in Step 3, we obtain a contradiction.

(ii) We choose the resolution $\pi: Z^{+} \rightarrow Z^{\prime}$ in Step 2 as in Lemma 2.6(2) (that corresponds to $\pi: Y \rightarrow X$ there). We have $K_{Z^{+}}=\pi^{*} K_{Z^{\prime}}$ over $U$. Then, by the base-point freeness of $m K_{Z^{\prime}}$, we can find a section $s \in H^{0}\left(Z^{+}, m K_{Z^{+}}\right)$such that $\left.s\right|_{\pi^{-1}(U)}$ has no zeros on $\pi^{-1}(U)$ (by shrinking $U$ if necessary). On the other hand, we have $Z_{0}^{+}=F^{+}+E$ over $U$ for a non-zero $\pi$-exceptional divisor $E$ (which may not be prime, but the precise form of $E_{\text {red }}$ is well known depending on the ADE type). We see that $F^{+} \cap E \neq \emptyset$.

Let $W \subset H^{0}\left(Z^{+}, m K_{Z^{+}}\right)$be the subspace generated by the sections obtained by the extensions from $\oplus_{j \in J} H^{0}\left(F_{j}^{+}, m K_{F_{j}^{+}}\right)$. If an irreducible component $F_{1}^{+}$of $F^{+}$intersects with $E$, we can see that every element of $W$ vanishes along $F_{1}^{+} \cap E_{\text {red }}$, at least (this holds for the extension in Theorem 2.4). Thus $s \notin W$. Then by a dimension-counting argument as in Step 3, we obtain a contradiction.

Step 5. We shall show that the normal variety $Z_{0}^{\prime}=X_{0}^{\prime}\left(=F^{\prime}\right.$, say) has canonical singularities at worst. Recall that $F^{+} \subset Z^{+}$is the strict transform of $F^{\prime}$. We know that $K_{F^{\prime}}$ is $\mathbb{Q}$-Cartier by Lemma 2.3(2). If $F^{\prime}$ has worse singularities than canonical ones, we have $K_{F^{+}} \sim_{\mathbb{Q}} \nu^{*} K_{F^{\prime}}+E-D$, where $\nu=\left.\pi\right|_{F^{+}}: F^{+} \rightarrow F^{\prime}$ is a resolution of singularities (we can suppose that $\nu$ is a logresolution of $F^{\prime}$ ), and where $E$ and $D$ are effective $\mathbb{Q}$-divisors on $F^{+}$, both $\nu$-exceptional and with $D \neq 0$.

Let us take an open subset $U^{\prime} \subset F^{\prime}$ such that $\nu: F^{+} \rightarrow F^{\prime}$ is biregular over $U^{\prime}$ and $\operatorname{codim}_{F^{\prime}}\left(F^{\prime} \backslash U^{\prime}\right) \geqslant 2$. For every $\sigma \in H^{0}\left(F^{+}, m F^{+}\right)$, the restriction $\left.\sigma\right|_{\nu^{-1}\left(U^{\prime}\right)}$ can be seen, via $\nu^{-1}\left(U^{\prime}\right) \cong U^{\prime}$, as an element of $H^{0}\left(U^{\prime}, m K_{F^{\prime}}\right)$. As $F^{\prime}$ is normal, we have $H^{0}\left(U^{\prime}, m K_{F^{\prime}}\right)=$ $H^{0}\left(F^{\prime}, m K_{F^{\prime}}\right)$. We thus obtain a natural injection $H^{0}\left(F^{+}, m K_{F^{+}}\right) \rightarrow H^{0}\left(F^{\prime}, m K_{F^{\prime}}\right)$ for any $m>0$ by "pushing down." Let us take $\tau \in H^{0}\left(F^{\prime}, m K_{F^{\prime}}\right)$ for a large and divisible $m>0$. The pull-back $\nu^{*} \tau \in H^{0}\left(F^{+}, \nu^{*}\left(m K_{F^{\prime}}\right)\right)\left(\cong H^{0}\left(F^{+}, \nu^{*}\left(m K_{F^{\prime}}\right)+m E\right)=H^{0}\left(F^{+}, m K_{F^{+}}+m D\right)\right)$ defines an element of $H^{0}\left(F^{+}, m K_{F^{+}}\right)$if and only if $\nu^{*} \tau \in H^{0}\left(F^{+}, \nu^{*}\left(m K_{F^{\prime}}\right)\right)$ vanishes along $m D$.

As $K_{X^{\prime}}$ is $f^{\prime}$-semi-ample, $\left.\left(m K_{X^{\prime}}\right)\right|_{F^{\prime}}=m K_{F^{\prime}}$ (see Lemma $\left.2.3(3)\right)$ is base-point free for $m$ large and sufficiently divisible. Thus there exists an element of $H^{0}\left(F^{\prime}, m K_{F^{\prime}}\right)$ which (does not vanish identically along $\nu(D)$ and hence) does not extend to an element of $H^{0}\left(F^{+}, m K_{F^{+}}\right)$. Then we have $h^{0}\left(F^{+}, m K_{F^{+}}\right)<h^{0}\left(F^{\prime}, m K_{F^{\prime}}\right)$. However, this is impossible, because on one hand, we have $h^{0}\left(F^{+}, m K_{F^{+}}\right)=P_{m}\left(F^{+}\right)=P_{m}\left(X_{y}\right)$ for general $y \in C$ by our assumption in Theorem 1.1 and on the other hand, we have $P_{m}\left(X_{y}\right)=h^{0}\left(X_{0}^{\prime}, m K_{X_{0}^{\prime}}\right)=h^{0}\left(F^{\prime}, m K_{F^{\prime}}\right)$ for any $y \in C$ by Lemma 2.3(3). 


\section{S. TAKAYAMA}

Step 6. We shall finally show that $F_{i} \subset \operatorname{SBs}\left(K_{X}\right)$ for $i \neq 1$. As $K_{Z}=\left.K_{X}\right|_{Z}$, we have $\left.\operatorname{SBs}\left(K_{Z}\right) \subset\left(\operatorname{SBs}\left(K_{X}\right)\right)\right|_{Z}$. Given that $Z_{0}=\bigcup_{i \in I} F_{i}$, it is enough to show that $\bigcup_{i \neq 1} F_{i} \subset$ $\operatorname{SBs}\left(K_{Z}\right)$.

Let $\varpi: \widetilde{Z} \rightarrow Z$ be a log-resolution of singularities such that the special fiber of $g \circ \varpi: \widetilde{Z} \rightarrow C$ is $\widetilde{Z}_{0}=\sum_{i \in I} \widetilde{F}_{i}+E$, where $\widetilde{F}_{i}$ is the strict transform of $F_{i}$ and $E$ is an effective divisor on $\widetilde{Z}$ whose support is $\varpi$-exceptional. We can take $\varpi$ such that $\widetilde{F}_{i} \cap \widetilde{F}_{j}=\emptyset$ for $i, j \in I$ and $i \neq j$.

We suppose to the contrary that there exist an integer $m>0$ and $s_{Z} \in H^{0}\left(Z, m K_{Z}\right)$ such that $\left.s_{Z}\right|_{F_{2}} \not \equiv 0$. Then we obtain $s_{\widetilde{Z}} \in H^{0}\left(\widetilde{Z}, m K_{\widetilde{Z}}\right)$ such that $\left.s_{\widetilde{Z}}\right|_{\widetilde{F}_{2}} \not \equiv 0$. On the other hand, by Theorem 2.4, we can extend every section $s_{1} \oplus 0 \in H^{0}\left(\widetilde{F}_{1}, m K_{\widetilde{F}_{1}}\right) \oplus\left(\oplus_{i \neq 1} H^{0}\left(\widetilde{F}_{i}, m K_{\widetilde{F}_{i}}\right)\right)$ with $s_{1} \in H^{0}\left(\widetilde{F}_{1}, m K_{\widetilde{F}_{1}}\right)$ to an element of $H^{0}\left(\widetilde{Z}, m K_{\widetilde{Z}}\right)$. As we already know that $\widetilde{F}_{1}$ is birational to $X_{0}^{\prime}$ and $P_{m}\left(\widetilde{F}_{1}\right)=P_{m}\left(X_{y}\right)$ for $y \in Y$ general, we can derive a contradiction by a dimension-counting argument, as before.

A weak converse of Theorem 1.1 holds in the following sense. The following argument, based on a uniruledness criterion in [HM07], also gives an alternative approach to Step 1 in the proof of Theorem 1.1.

Proposition 2.5. Suppose that $f: X \rightarrow Y$ is weakly semi-stable and that there exists a good minimal model $f^{\prime}: X^{\prime} \rightarrow Y$ of $X$ over $Y$. Let $0 \in Y$ be a point, and let $X_{0}^{\prime}=\bigcup_{\ell \in L} G_{\ell}^{\prime}$ be the decomposition into irreducible components of the fiber of $f^{\prime}$ over 0 . Suppose $\kappa\left(G_{\ell}^{\prime}\right) \geqslant 0$ for any $\ell \in L$.

Then the fiber of $f$ over 0 is $X_{0}=\bigcup_{\ell \in L} G_{\ell} \cup \bigcup_{\lambda \in \Lambda} F_{\lambda}$, with irreducible components $G_{\ell}$ birational to $G_{\ell}^{\prime}$ by the birational map $\varphi: X \rightarrow X^{\prime}$ and uniruled components $F_{\lambda}$.

Proof. We take a general smooth curve $C \subset Y$ passing through 0 as in Lemma 2.3(1). Let $g: Z:=X \times{ }_{Y} C \rightarrow C$ and $g^{\prime}: Z^{\prime}:=X^{\prime} \times_{Y} C \rightarrow C$ be the induced morphisms. We take a common resolution of singularities $Z^{+}$of $Z$ and $Z^{\prime}$ sitting in the following commutative diagram:

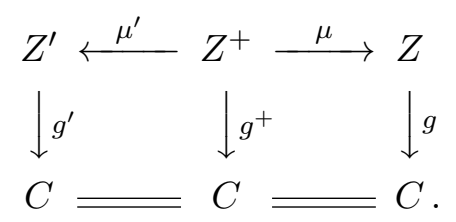

Let $G_{\ell}^{+} \subset Z^{+}$be the strict transform of $G_{\ell}^{\prime}$. If $\operatorname{dim} \mu\left(G_{\ell}^{+}\right)<\operatorname{dim} G_{\ell}^{+}$, then $G_{\ell}^{+}$is uniruled by [HM07, Corollary 1.5], which is impossible. Hence $\operatorname{dim} \mu\left(G_{\ell}^{+}\right)=\operatorname{dim} G_{\ell}^{+}$. As one can choose $\mu$ such that it is biregular on a Zariski-open subset $U \subset Z$ with $\operatorname{codim}_{Z}(Z \backslash U) \geqslant 2$, the morphism $G_{\ell}^{+} \rightarrow \mu\left(G_{\ell}^{+}\right)$is birational. We let $F_{\ell}=\mu\left(G_{\ell}^{+}\right)$. These $F_{\ell}$ are mutually different divisors in $Z$. In fact, if $F_{1}=F_{2}$, for example, we have $\mu^{-1}\left(F_{1} \cap U\right)=\mu^{-1}\left(F_{2} \cap U\right)$. As $\mu^{-1}\left(F_{i} \cap U\right)$ is a non-empty open subset of $G_{i}^{+}$, we can conclude that $G_{1}^{+}=G_{2}^{+}$. We also note that $\bigcup_{\ell \in L} F_{\ell} \subset X_{0}$. We take an irreducible component $F$ of $X_{0}$ other than $G_{\ell}=\varphi_{*}\left(G_{\ell}^{\prime}\right)$ s and let $F^{+} \subset Z^{+}$be the strict transform. Then $\mu^{\prime}\left(F^{+}\right) \subset X_{0}^{+}$, but it is different from $G_{\ell}$. Hence $F^{+}$is $\mu^{\prime}$-exceptional, and therefore $F^{+}$(as well as $F$ ) is uniruled, again by [HM07, Corollary 1.5].

It can happen that $f: X \rightarrow Y$ is semi-stable and good minimal but that $X_{0}=\bigcup_{i \in I} F_{i}$ with $P_{m}\left(X_{y}\right)>\sum_{i \in I} P_{m}\left(F_{i}\right)$ for all $m>0$ and general $y \in Y$. This happens, for example, when $f$ is a relatively minimal elliptic fibration over a curve and $X_{0}=\bigcup_{i \in I} F_{i}$ is a cycle of projective lines $\mathbb{P}^{1}$ (a degeneration of type $\mathrm{I}_{b}$ in Kodaira's classification). 


\section{FILLING-IN PROBLEM AND DEGENERATIONS OF VARIETIES}

We close this section by proving a general lemma, which can be seen as a very weak version of simultaneous crepant resolutions of ADE-type surface singularities.

Lemma 2.6. Let $X$ be an $n$-dimensional quasi-projective variety with canonical singularities, and let $B \subset X$ be an irreducible subvariety. Suppose $\operatorname{dim} B=n-2$ and that $B$ is an irreducible component of Sing $X$. Let $|H|$ be a very ample linear system of Cartier divisors on $X$, and let $S=H_{1} \cap \cdots \cap H_{n-2}$ be a surface obtained as a complete intersection of $n-2$ general members in $|H|$.

(1) There exists a Zariski-open subset $U \subset X$ which intersects $B$ and has the property that $S \cap U$ has ADE-type singularities at $B \cap S \cap U$ and its ADE-type is independent of $S$ and the points in $B \cap S \cap U$. (The intersection $B \cap S \cap U$ may be not one point but a finite number of points.)

(2) There exists a log-resolution of singularities $\pi: Y \rightarrow X$ such that the induced morphism $\pi^{-1}(S \cap U) \rightarrow S \cap U$ is the minimal resolution of the ADE-type singularities $(S \cap U, B \cap S \cap U)$, and $K_{Z}=\pi^{*} K_{X}$ over $U$, that is, $K_{\pi^{-1}(U)}=\pi^{*} K_{U}$, where $U \subset X$ is a Zariski-open set as in item (1).

Proof. Let $\alpha: Z \rightarrow X$ be a log-resolution of singularities such that $\alpha$ is an isomorphism over $X \backslash \operatorname{Sing} X$, and let $\left\{E_{\lambda}\right\}_{\lambda \in \Lambda}$ be the set of all $\alpha$-exceptional prime divisors. We write $\mathcal{I}_{B} \cdot \mathcal{O}_{Z}=$ $\mathcal{O}_{Z}\left(-\sum_{i \in I} m_{i} E_{i}\right)$ for a subset $I \subset \Lambda$ with integers $m_{i}>0$, where $\mathcal{I}_{B} \cdot \mathcal{O}_{Z}$ (sometimes written as $\alpha^{-1} \mathcal{I}_{B} \cdot \mathcal{O}_{Z}$ ) is the inverse-image ideal sheaf of $\mathcal{I}_{B} \subset \mathcal{O}_{X}$ (see [Har77, II, Definition following Example 7.12.1]). We can write $K_{Z}={ }_{\mathbb{Q}} \alpha^{*} K_{X}+\sum a_{\lambda} E_{\lambda}$ with $a_{\lambda} \in \mathbb{Q} \geqslant 0$.

We consider a Zariski-open subset $U \subset X$ satisfying the following properties: $B \cap U \neq \emptyset$, $B \cap U$ is smooth, $B \cap U=\operatorname{Sing} U, U \cap \alpha\left(E_{\lambda}\right)=\emptyset$ for any $\lambda \in \Lambda$ with $\alpha\left(E_{\lambda}\right) \neq B$, and $U$ is Gorenstein (note that a canonical singularity is Gorenstein in codimension 2). Exceptional divisors $E_{\lambda}$ with $\alpha\left(E_{\lambda}\right) \neq B$ are actually irrelevant in our argument. Hence, we will assume $I=\Lambda$ (there might be an $i \in I$ such that $\alpha\left(E_{i}\right) \neq B$ ). We can also suppose, to simplify our argument, that $U$ is affine, $K_{U}$ is a principal divisor, and $|H|_{\mid U}$ is a linear system of principal divisors. We can further suppose that every morphism $E_{i} \rightarrow B$ over $U$ (if $E_{i} \cap \alpha^{-1}(U) \neq \emptyset$ ) is surjective, smooth of relative dimension 1. (The morphism $\sum E_{i} \rightarrow B$ over $U$ may be called a "relative normal crossing," $E_{i} \cap E_{j} \rightarrow B$ is finite étale, and $E_{i} \cap E_{j} \cap E_{k}=\emptyset$ over $U$. But we do not know whether $E_{i} \rightarrow B$ has connected fibers over $U$.)

As $S$ is general, $\widetilde{S}=\alpha^{-1}(S)$ is smooth by Bertini's theorem $\left(\alpha^{-1}(S)\right.$ is in fact a schemetheoretic complete intersection of $n-2$ general members of $\left.\pi^{*}|H|\right)$. It is known that $S$ is Gorenstein canonical and hence has ADE-type singularities along $B \cap S$ (we refer to [Ish14, Corollary 7.3.2] in general). Moreover, by adjunction, $K_{\widetilde{S}}=\alpha_{S}^{*} K_{S}+\left.\sum a_{i} E_{i}\right|_{\widetilde{S}}$ as in the proof of [Ish14, Proposition 7.3.1]. Here we let $\alpha_{S}: \widetilde{S} \rightarrow S$ be the induced morphism. We actually have $K_{S}=\left.K_{X}\right|_{S}$ and $K_{\widetilde{S}}=\left.K_{Z}\right|_{\widetilde{S}}$ over $U$. By Bertini's theorem, we can suppose that "over $U$," every $\left.E_{i}\right|_{\widetilde{S}}$ is non-empty and smooth (if $E_{i} \cap \alpha^{-1}(U) \neq \emptyset$ ) but may not be irreducible. We take an arbitrary point $b \in B \cap S \cap U$. We look at the collection $\left\{\alpha_{S}^{-1}(b) \cap E_{i} ; i \in I\right\}$ of smooth $\alpha_{S^{-}}$exceptional curves in $\widetilde{S}$ whose connected components are all isomorphic to $\mathbb{P}^{1}$ and contracted to the point $b$. We especially look at the set $\left\{\left(\alpha_{S}^{-1}(b) \cap E_{i}, m_{i}\right) ; i \in I\right.$ with $\left.a_{i}=0\right\}$. We recall that $\left.\left(\mathcal{I}_{B} \cdot \mathcal{O}_{Z}\right)\right|_{\widetilde{S}}=\mathcal{O}_{\widetilde{S}}\left(-\left.\sum m_{i} E_{i}\right|_{\widetilde{S}}\right)$. The induced resolution $\alpha_{S}: \widetilde{S} \rightarrow S$ may not be the minimal one, but, thanks to the classification of the ADE-type singularities ([Ish14, Theorem 6.5.1], [KM98, Theorem 4.20]), the set of $\alpha_{S}$-exceptional curves with the null $K_{S}$-discrepancy, that is, $a_{i}=0$, with its multiplicity $m_{i}$ characterizes the ADE-type of the singularity $(S, b)$. These curves (which 


\section{S. TAKAYAMA}

are given by $(X, B))$ are independent of the general $S$ and the point $b \in B \cap S \cap U$. This is our first assertion (1).

For assertion (2), we run a $K_{Z}$-MMP for $\alpha: Z \rightarrow X$ over $X$, where $\alpha: Z \rightarrow X$ is a logresolution as above. We can run it because general fibers of $\alpha$ (it is a point) have good minimal models. By replacing $U$ in statement (1) with a smaller Zariski-open subset, we can suppose that we do not have flipping contractions over $U$. There may be some divisorial contractions. The image of the contracted divisors must be $B$ over $U$ (we could suppose $\alpha: Z \rightarrow X$ to be isomorphic over $U \backslash B$ ). Let $\pi: Y \rightarrow X$ be a minimal model. Then $Y$ has terminal singularities. In particular, $Y$ is smooth in codimension 2, and hence by replacing $U$ with a smaller Zariski-open subset (respecting $B$ ), we may suppose that $Y$ is smooth over $U$.

We now claim that $K_{Y}=\pi^{*} K_{X}$ over $U$. Otherwise, we would have $K_{Y}=\pi^{*} K_{X}+F$ over $U$ with all irreducible components of $F$ non-zero effective, $\pi$-exceptional, and $\pi(F)=B$ ( $K_{X}=0$ over $U$, in fact). By taking $S \subset X$ general and letting $\pi_{S}: T:=\pi^{-1}(S) \rightarrow S$, this would give a resolution of singularities of ADE-type singularities $(S, B)$ which is not crepant; $K_{T}=\pi_{S}^{*} K_{S}+\left.F\right|_{T}$ and $\left.F\right|_{T}$ is non-zero effective over $S \cap U$. Hence, $K_{T}$ is not $\pi_{S}$-nef [Ish14, Theorem 6.5.1]. We note that $K_{T}=\left.K_{Y}\right|_{T}$ over $U$. This implies that $K_{Y}$ is not $\pi$-nef, which leads to a contradiction. We also obtain that $K_{T}=\pi_{S}^{*} K_{S}$, and in particular $\pi_{S}: T \rightarrow S$ is the minimal resolution of $S$ [Ish14, Theorem 6.5.1].

We next show that $\pi: Y \rightarrow X$ gives a log-resolution of $B$ over $U$; namely, we show that the ideal $\mathcal{I}_{B} \cdot \mathcal{O}_{Z}$ defines a divisor with simple normal crossing support over $U$. The double dual $\left(\mathcal{I}_{B} \cdot \mathcal{O}_{Y}\right)^{* *} \subset \mathcal{O}_{Y}$ is a reflexive sheaf of rank 1 on $Y$, and hence can be expressed as $\mathcal{O}_{Y}\left(-\sum_{j} m_{j} F_{j}\right)$ for some prime Weil divisor $F_{j}$ on $Y$ and integers $m_{j}>0$. Moreover, on $W:=$ $\pi^{-1}(U)$, which is smooth, $\left(\mathcal{I}_{B} \cdot \mathcal{O}_{Y}\right)^{* *}$ is invertible. We can suppose $\pi\left(F_{j}\right)=B$ if $F_{j} \cap W \neq \emptyset$. We have an exact sequence

$$
0 \rightarrow \mathcal{I}_{B} \cdot \mathcal{O}_{Y} \rightarrow\left(\mathcal{I}_{B} \cdot \mathcal{O}_{Y}\right)^{* *} \rightarrow Q \rightarrow 0,
$$

where the left injection is the natural one and $Q$ is the cokernel with $\operatorname{dim} \operatorname{Supp} Q \leqslant n-2$. We consider the minimal resolution $\pi_{S}: T \rightarrow S$ as above.

If $\pi(\operatorname{Supp} Q)=B$, then $\left.\left(\mathcal{I}_{B} \cdot \mathcal{O}_{Y}\right)\right|_{T} \subset \mathcal{O}_{T}$ is not exactly an ideal sheaf of an effective divisor, because of an exact sequence

$$
\left.\left.\left(\mathcal{I}_{B} \cdot \mathcal{O}_{Y}\right)\right|_{T} \rightarrow \mathcal{O}_{T}\left(-\left.\sum_{j} m_{j} F_{j}\right|_{T}\right) \rightarrow Q\right|_{T} \rightarrow 0
$$

where $\left.Q\right|_{T}$ is non-zero and $\left.\operatorname{dim} \operatorname{Supp} Q\right|_{T}=0$. This contradicts the fact that $\left.\left(\mathcal{I}_{B} \cdot \mathcal{O}_{Y}\right)\right|_{T}$ corresponds to the exceptional divisor of $\pi_{S}: T \rightarrow S$ (given by $\pi_{S}^{-1} \mathfrak{m}_{b} \cdot \mathcal{O}_{T}$ for every point $b \in B \cap S \cap U$ ). Hence, we have $Q=0$ over $U$ (by shrinking $U$ ) and therefore $\mathcal{I}_{B} \cdot \mathcal{O}_{Y} \cong \mathcal{O}_{Y}\left(-\sum_{j} m_{j} F_{j}\right.$ ) on $W$.

We next show that the divisor $\sum F_{j}$ is normal crossing over $U$, by shrinking $U$. If not, the ideal sheaf $\left.\left(\mathcal{I}_{B} \cdot \mathcal{O}_{Y}\right)\right|_{T} \subset \mathcal{O}_{T}$ cannot correspond to an effective divisor with normal crossing support on $T$. However, we already know that the exceptional curves of the minimal resolution $\pi_{S}: T \rightarrow S$ have normal crossing support. Hence, $\sum F_{j}$ is normal crossing over $U$.

Finally, we show that $\sum F_{j}$ is simple normal crossing over $U$; that is, every $F_{j}$ is smooth on $W$. It is enough to show that every $F_{j}$ has no self-intersection on $W$. If it has, we then have an exceptional curve of $\pi_{S}: T \rightarrow S$ with self-intersection. But this is not the case. (We do not know whether every morphism $F_{j} \rightarrow B$ over $U$ has connected fiber.)

The above $\pi: Y \rightarrow X$ may not be a resolution of $X$. We then modify $Y$ further along $Y \backslash W$ and obtain a log-resolution as in assertion (2). 


\section{FILLING-IN PROBLEM AND DEGENERATIONS OF VARIETIES}

\section{A filling-in problem, I}

We shall discuss the filling-in problem as stated in Theorem 1.4 in this and the next sections. This section is devoted to some preliminaries.

SETup 3.1. (1) Let $X$ be a normal quasi-projective variety and $C$ a smooth affine curve with a special point $0 \in C$, and let $f: X \rightarrow C$ be a projective surjective morphism with connected fibers. In particular, $K_{C}=\mathcal{O}_{C}$. Let $X_{0}:=f^{*}(0)=\sum_{i \in I} n_{i} F_{i}$ be the primary decomposition of the special/central fiber, which may be non-reduced. The symbol $t$ will also stand for a local coordinate of $C$ centered at 0 . We may replace $C$ by a smaller neighborhood of 0 .

(2) Suppose that $X^{o}:=X \backslash f^{-1}(0)$ is smooth, that $f$ is smooth on $X^{o}$ and that $X_{t}:=f^{-1}(t)$ has semi-ample canonical bundle $K_{X_{t}}$ for any $t \in C^{o}:=C \backslash\{0\}$. As every general fiber $X_{t}$ is a good minimal model, a good minimal model of $X$ over $C$ exists by [HX13, Theorem 2.12] (see also [Fuj16a, Theorem 3.3]).

Wang [Wan03, Proposition 1.3] proved the implication $(1) \Rightarrow(2)$ of Theorem 1.4 and posed the converse as a problem. His results are roughly summarized as follows.

Theorem 3.2 (Wang). Let $f: X \rightarrow C$ be as in Setup 3.1 with (however) $C=\Delta$ a disc in $\mathbb{C}$.

(1) [Wan03, Theorem 1.1(2)]. Suppose that $f: X \rightarrow C$ is semi-stable with $X_{0}=\sum_{i \in I} F_{i}$ and $p_{g}\left(X_{t}\right) \neq 0$ for $t \in C^{o}$, where $p_{g}$ stands for the geometric genus. Then $g_{H}\left(=\omega_{1}\right.$ in Definition 1.3) is incomplete at $0 \in C$ if and only if $p_{g}\left(X_{t}\right)=\sum_{i \in I} p_{g}\left(F_{i}\right)$ for $t \in C^{o}$.

(2) [Wan03, Theorem 1.1(3), Corollary 2.4]. If $X_{0}$ has Gorenstein canonical singularities, then $0 \in C$ is at finite $g_{1}$ distance; that is, $\omega_{1}$ is incomplete at $0 \in C\left(\right.$ if $\left.f_{*} K_{X^{o}} / C^{o} \neq 0\right)$.

(3) [Wan03, Proposition 1.3]. If $X_{0}$ has canonical singularities and $K_{X_{t}}$ semi-ample for any $t \neq 0$, then $0 \in C$ is at finite $g_{m}\left(=\omega_{m, D}\right.$ in Definition 1.3) distance for all $m$ such that $g_{m}$ is defined.

In [Wan03, Propositions 1.2 and 1.3], assumptions on the singularities of the total space $X$ and on the semi-ampleness of the canonical divisor $K_{X_{0}}$ of the central fiber $X_{0}$ are missing. In the course of our preparations toward Theorem 1.4, we will recall and complete the proof of [Wan03, Proposition 1.3] as Proposition 3.10, under correct assumptions.

To obtain the converse implication $(2) \Rightarrow(1)$ of Theorem 1.4, following the proposal in [Wan03, §3], we look at a condition on the invariance of plurigenera type defined as follows.

Definition 3.3. Let $f: X \rightarrow C$ and $X_{0}=\sum_{i \in I} n_{i} F_{i}$ be as in Setup 3.1. Let $m>0$ be a positive integer. We say that $f: X \rightarrow C$ has the property $\left(P_{m}\right)$ if $\sum_{i \in I} P_{m}\left(F_{i}\right)=P_{m}\left(X_{t}\right)$ for $t \in C^{o}$ general.

We shall state a few basic properties and remarks related to this property $\left(P_{m}\right)$. We know, for example by [Tak07, Theorem 1.1], that if $X_{0}$ has canonical singularities at worst, then $f$ has the property $\left(P_{m}\right)$ for any $m>0$. If $f: X \rightarrow C$ is an elliptic fibration of a smooth surface $X$ with a multiple fiber $X_{0}=n_{1} F_{1}$ for a smooth elliptic curve $F_{1}$, then $f$ has the property $\left(P_{m}\right)$, but $X_{0}$ can be non-reduced.

Lemma 3.4. Let $f: X \rightarrow C$ and $f^{\prime}: X^{\prime} \rightarrow C^{\prime}$ be morphisms fitting Setup 3.1 with special points 


\section{S. TAKAYAMA}

$0 \in C$ and $0^{\prime} \in C^{\prime}$. Suppose that we have the following commutative diagram:

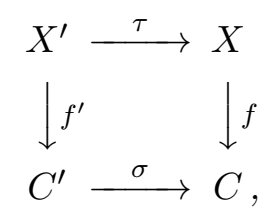

where $\sigma: C^{\prime} \rightarrow C$ is a finite morphism with $\sigma\left(0^{\prime}\right)=0$, and where the morphism $\tau: X^{\prime} \rightarrow X$ is generically finite and $X_{s}^{\prime} \rightarrow X_{\sigma(s)}$ is birational if $s \in C^{\prime}$ is general. Let $m>0$ be an integer.

(1) If $f$ has the property $\left(P_{m}\right)$, then so does $f^{\prime}$.

(2) Suppose $C^{\prime}=C$, that $\sigma: C^{\prime} \rightarrow C$ is the identity map (then $\tau: X^{\prime} \rightarrow X$ is birational), and that $X$ has canonical singularities at worst. Then $f$ has the property $\left(P_{m}\right)$ if and only if $f^{\prime}$ does.

Proof. We let $X_{0^{\prime}}^{\prime}:=f^{\prime *}\left(0^{\prime}\right)=\sum_{j \in J} \ell_{j} G_{j}$ be the primary decomposition of the special fiber. For any general $s \in C^{\prime}$, we have the identity $P_{m}\left(X_{s}^{\prime}\right)=P_{m}\left(X_{\sigma(s)}\right)$ and an inequality $P_{m}\left(X_{s}^{\prime}\right) \geqslant$ $\sum_{j \in J} P_{m}\left(G_{j}\right)$ by [Tak07, Theorem 1.2].

(1) For every component $F_{i}$ of $X_{0}$, there exists a component $G_{j}$ of $X_{0^{\prime}}^{\prime}$ such that $\tau: G_{j} \rightarrow F_{i}$ is surjective and hence generically finite. To see $P_{m}\left(G_{j}\right) \geqslant P_{m}\left(F_{i}\right)$ when $\tau: G_{j} \rightarrow F_{i}$ is generically finite, it is enough to work on smooth models, in which case the pull-back of pluricanonical forms is injective and yields the expected inequality. Thus we can deduce $\sum_{j \in J} P_{m}\left(G_{j}\right) \geqslant \sum_{i \in I} P_{m}\left(F_{i}\right)$ and our first assertion.

(2) Let $G_{j}$ be a $\tau$-exceptional component. Then it is uniruled by [HM07, Corollary 1.5], and in particular $P_{m}\left(G_{j}\right)=0$ (we can allow "divisorial log terminal" singularities of $X$ to apply [HM07, Corollary 1.5]). Let $G_{j}$ be a non- $\tau$-exceptional component. By virtue of Zariski's main theorem, $\tau$ is isomorphic in codimension 1 near $G_{j}$, and, therefore, $\tau$ induces a birational map to a component $F_{i}$. Hence, we obtain $\sum_{j \in J} P_{m}\left(G_{j}\right)=\sum_{i \in I} P_{m}\left(F_{i}\right)$ and our second assertion.

We can generalize Theorem 1.1 slightly if the base is a curve. We do not assume $f$ to be weakly semi-stable, and, in particular, its fibers can be non-reduced, unlike in Theorem 1.1.

Corollary 3.5. Let $f: X \rightarrow C$ be as in Setup 3.1, and let $X_{0}:=f^{*}(0)=\sum_{i \in I} n_{i} F_{i}$. Suppose that $f$ has the property $\left(P_{m}\right)$ for every large and divisible integer $m>0$. Then there exists an irreducible component of $X_{0}$, say $F_{1}$, such that $P_{m}\left(F_{1}\right)=P_{m}\left(X_{t}\right)$ holds for every integer $m>0$ and a general point $t \in C$, and other components $F_{i}(i \in I \backslash\{1\})$ are uniruled (if $\left.|I| \geqslant 2\right)$.

Proof. By Lemma 3.4, a semi-stable reduction $\widetilde{f}: \widetilde{X} \rightarrow \widetilde{C}$ of $f$ has the property $\left(P_{m}\right)$ for every large and divisible integer $m>0$. Then by Theorem 1.1 for $\widetilde{f}$, the special fiber of $\widetilde{f}$ has a unique component $\widetilde{F}_{1}$ such that $P_{m}\left(\widetilde{F}_{1}\right)=P_{m}\left(\widetilde{X}_{s}\right)$ for $s \in \widetilde{C}$ general and other components are uniruled. Because of the existence of the dominant morphism $\widetilde{X} \rightarrow X$, we can suppose that all $F_{i}(i \in I \backslash\{1\})$ are uniruled after relabeling. We can then easily deduce our assertion.

We add a remark for future reference. If we further take a good minimal model $\widetilde{f}^{\prime}: \widetilde{X}^{\prime} \rightarrow \widetilde{C}$ of $\widetilde{X}$ over $\widetilde{C}$, we obtain that the special fiber $\widetilde{X}_{\widetilde{0}}^{\prime}$ (where $\widetilde{0} \in \widetilde{C}$ is a point mapped to $0 \in C$ ) has canonical singularities and $K_{\widetilde{X}_{\tilde{0}}^{\prime}}$ is semi-ample.

We prepare some lemmas, which can be stated in a slightly more general setting than Setup 3.1. We refer to [KM98, Definition 2.34] for the singularities of pairs such as Kawamata log terminal (klt), purely log terminal (plt) and log canonical (lc). 


\section{FILLING-IN PROBLEM AND DEGENERATIONS OF VARIETIES}

Lemma 3.6. Let $X$ be a normal variety such that the pair $(X, 0)$ has klt singularities, and let $|L|$ be a base-point free linear system of Cartier divisors on $X$.

(1) Let $0<\varepsilon<1$ be a rational number. Then the pair $(X, \varepsilon D)$ has klt singularities at worst for general $D \in|L|$.

(2) Suppose further that there exists a Cartier divisor $X_{0} \subset X$ such that $\left(X, X_{0}\right)$ is lc. Then $\left(X, D+X_{0}\right)$ is lc for general $D \in|L|$.

Proof. Let $D \in|L|$ be a general member.

(1) By [KM98, Lemma 5.17(1)], every connected component of $D$ is normal and has klt singularities. By an inversion of adjunction [KM98, Theorem $5.50(1)$ ], the pair $(X, D)$ is plt near $D$ and of course klt on $X \backslash D$. We have applied [KM98, Theorem 5.50(1)] with $S$ a connected component of $D$ and $B=0$. Then $(X, \varepsilon D)$ is klt on $X$ by decreasing the total discrepancy a bit.

(2) By $[\mathrm{KM} 98$, Lemma $5.17(1)]$, we see that $D$ is klt and $\left(D,\left.X_{0}\right|_{D}\right)$ is lc. Then by [KM98, Theorem 5.50], the pair $\left(X, D+X_{0}\right)$ is lc near $D$ and hence lc on $X$.

Lemma 3.7. Let $X$ be a normal variety such that the pair $(X, 0)$ has klt singularities. Suppose that $K_{X}$ is semi-ample, and take an integer $m>0$ such that $m K_{X}$ is Cartier and the linear system $\left|m K_{X}\right|$ is base-point free. Let $D \in\left|m K_{X}\right|$ be a general member, let $Y:=\operatorname{Spec}\left(\oplus_{i=0}^{m-1} \omega_{X}^{[-i]}\right)$, and let $\pi: \rightarrow X$ be the cyclic cover obtained by taking the $m$ th root of $D$ (see Item 3.8). Then $Y$ has canonical singularities at worst and $K_{Y}=\pi^{*}\left(m K_{X}\right)$, which in particular implies that $K_{Y}$ is Cartier and the linear system $\left|K_{Y}\right|$ is base-point free. Moreover, given a Cartier divisor $X_{0} \subset X$ such that the pair $\left(X, X_{0}\right)$ is $l c$, one can take $D \in\left|m K_{X}\right|$ such that $\left(Y, Y_{0}\right)$ is lc, where $Y_{0}=\pi^{*} X_{0}$, and that $D$ does not contain any irreducible components of the singular locus Sing $X_{0}$ of $X_{0}$. We will refer to this property as " $D \in\left|m K_{X}\right|$ is $\left(X, X_{0}\right)$-general."

3.8. Let us recall the definition of the cyclic covering $\pi: Y \rightarrow X$ (or $\pi_{D}: Y \rightarrow X$ ) associated with $D$ in the setting of Lemma 3.7. This construction itself has nothing to do with $K_{X}$ and can be carried out for any torsion-free sheaf of rank 1 . We let $\omega_{X}^{[i]}=\mathcal{O}_{X}\left(i K_{X}\right)$ for every integer $i$; equivalently, $\omega_{X}^{[i]}$ is the double dual of $\omega_{X}^{\otimes i}$ and $\omega_{X}^{[-i]}$ is the dual of $\omega_{X}^{[i]}$ for $i \geqslant 0$. Then $Y=\operatorname{Spec}\left(\oplus_{i=0}^{m-1} \omega_{X}^{[-i]}\right) \rightarrow X$ is given by the following $\mathcal{O}_{X}$-algebra structure on the sheaf $\mathcal{A}=\oplus_{i=0}^{m-1} \omega_{X}^{[-i]}$. We take a section $s \in H^{0}\left(X, \omega_{X}^{[m]}\right)$ whose zero divisor is $D$. We regard $s$ as an element of $\operatorname{Hom}\left(\mathcal{O}_{X}, \omega_{X}^{[m]}\right)$ and then put an $\mathcal{O}_{X}$-algebra structure on $\mathcal{A}$ with multiplication given (if $i+j \geqslant m$ ) by

$$
\omega_{X}^{[-i]} \otimes \omega_{X}^{[-j]} \rightarrow \omega_{X}^{[-i-j]} \otimes \mathcal{O}_{X} \stackrel{i d \otimes s}{\longrightarrow} \omega_{X}^{[-i-j]} \otimes \omega_{X}^{[m]} \cong \omega_{X}^{[-i-j+m]} .
$$

It has the property that $\pi_{*} \mathcal{O}_{Y}=\oplus_{i=0}^{m-1} \omega_{X}^{[-i]}$. (To be more precise, if we let $\mu: \tilde{Y} \rightarrow Y$ be the normalization of $Y$ and $\tilde{\pi}=\pi \circ \mu: \widetilde{Y} \rightarrow X$ be the induced finite morphism, then we actually have $\widetilde{\pi}_{*} \mathcal{O}_{\widetilde{Y}}=\mathcal{A}$ and hence $\widetilde{Y}=\operatorname{Spec} \mathcal{A}(=Y)$ as in [EV92, Claim 3.10]. The point is that $\mathcal{A}$ is reflexive and that we can suppose $\operatorname{Sing} D \subset \operatorname{Sing} X$ for $D \in\left|m K_{X}\right|$ general.)

As soon as $K_{Y}=\pi^{*}\left(m K_{X}\right)$ is established, we then have $\pi_{*} \mathcal{O}_{Y}\left(K_{Y}\right)=\pi_{*}\left(\pi^{*} \mathcal{O}_{X}\left(m K_{X}\right)\right)=$ $\mathcal{O}_{X}\left(m K_{X}\right) \otimes \pi_{*} \mathcal{O}_{Y}=\oplus_{i=1}^{m} \mathcal{O}_{X}\left(i K_{X}\right)=\oplus_{i=1}^{m} \omega_{X}^{[i]}$ and

$$
\pi_{*} \mathcal{O}_{Y}\left(\ell K_{Y}\right)=\oplus_{i=1}^{m} \mathcal{O}_{X}\left((m(\ell-1)+i) K_{X}\right)
$$

in general for every integer $\ell>0$. We refer to [KM98, Definitions 2.52 and 5.19] and [EV92, §3] for more details. 


\section{S. TAKAYAMA}

Proof of Lemma 3.7. We set $D_{Y}=\left(\pi^{*} D\right)_{\text {red }}=\pi^{-1}(D)$. Then $\pi^{*} D=m D_{Y}$. We also have the Hurwitz-type formulas

$$
K_{Y}+D_{Y}=\pi^{*}\left(K_{X}+D\right), \quad K_{Y}=\pi^{*} K_{X}+(m-1) D_{Y}, \quad K_{Y}=\pi^{*}\left(K_{X}+\frac{m-1}{m} D\right) .
$$

Every relation initially holds on $\pi^{-1}\left(X_{\text {reg }}\right)$ and then holds on $Y$. In particular, $K_{Y}=\pi^{*}\left(m K_{X}\right)$. As the pair $(X,((m-1) / m) D)$ is klt by Lemma 3.6, the adjunction formula $K_{Y}=\pi^{*}\left(K_{X}+\right.$ $((m-1) / m) D)$ implies that the pair $(Y, 0)$ is klt by [KM98, Proposition 5.20(4)]. As $K_{Y}$ is Cartier, $Y$ in fact has canonical singularities at worst.

By Lemma 3.6, the pair $\left(X, D+X_{0}\right)$ is lc and, in particular, $\left(X,((m-1) / m) D+X_{0}\right)$ is lc. We know $K_{Y}+Y_{0}=\pi^{*}\left(K_{X}+((m-1) / m) D+X_{0}\right)$. Thus by [KM98, Proposition 5.20(4)], the pair $\left(Y, Y_{0}\right)$ is lc.

The following lemma is a variant of the inversion of adjunction [KM98, Theorem 5.50] and must be well known to experts. We refer to [Kol13, § 5.2] for semi-log-canonical singularities.

Lemma 3.9. Let $X$ be a normal variety with $\mathbb{Q}$-Cartier canonical divisor $K_{X}$, and let $S \subset X$ be an effective Cartier divisor. If $S$ is semi-log-canonical, then the pair $(X, S)$ is lc near $S$ (that is, lc on a neighborhood of $S$ ).

Proof. By definition [Kol13, Definition-Lemma 5.10], the divisor $S$ is demi-normal (see [Kol13, Definition 5.1] for the definition). We know $\left.\left(K_{X}+S\right)\right|_{S}=K_{S}$ by [Kol13, Proposition 4.5(4),(5)]. Let $\pi: \bar{S} \rightarrow S$ be the normalization, and let $\bar{D} \subset \bar{S}$ be the conductor subscheme (see [Kol13, 5.2, p. 189] for the definition). We have $\pi^{*} K_{S} \sim_{\mathbb{Q}} K_{\bar{S}}+\bar{D}$ by [Kol13, (5.7.5)]. On the other hand, by $\left[\right.$ Kol13, (4.2.9)], there exists an effective $\mathbb{Q}$-divisor $\operatorname{Diff}_{\bar{S}}(0)$ on $\bar{S}$ such that $\pi^{*}\left(\left.\left(K_{S}+S\right)\right|_{S}\right) \sim_{\mathbb{Q}}$ $K_{\bar{S}}+\operatorname{Diff}_{\bar{S}}(0)$ holds. As $\left.\left(K_{X}+S\right)\right|_{S}=K_{S}$, we see that $\operatorname{Diff}_{\bar{S}}(0) \sim_{\mathbb{Q}} \bar{D}$. In fact, by the uniqueness property in $[K o l 13,(4.2 .7)]$ and $[K o l 13,(5.7 .4)]$, we have $\operatorname{Diff}_{\bar{S}}(0)=\bar{D}$. By definition $[$ Kol13, Definition-Lemma 5.10], the divisor $S$ is semi-log-canonical if and only if $(\bar{S}, \bar{D})$ is lc (at least in our setting). Thus $\left(\bar{S}, \operatorname{Diff}_{\bar{S}}(0)\right)$ is lc, and then $(X, S+0)$ is lc near $S$ by the inversion of adjunction [Kol13, Theorem 4.9(2)].

We now recall and complete the proof of [Wan03, Proposition 1.3], that is, the implication $(1) \Rightarrow(2)$ of Theorem 1.4

Proposition 3.10 ([Wan03, Proposition 1.3]). Suppose in Setup 3.1 that $X_{0}$ has canonical singularities. Then, after possibly a finite base change and a birational modification along the special fiber, $g_{m, D}$ is incomplete at $0 \in C$ for every large and divisible integer $m$ and every general $D \in\left|m K_{X / C}\right|$. If we further suppose that $K_{X_{0}}$ is semi-ample, then we do not need to take finite base changes nor birational modifications along the special fiber.

Proof. We note that $X$ has canonical singularities at worst by [Kaw99]. If $K_{X_{0}}$ is semi-ample, then $f: X \rightarrow C$ is a good minimal model. If $K_{X_{0}}$ is not semi-ample, we change the model as follows. We note that $f$ has the property $\left(P_{k}\right)$ for every $k>0$ by [Tak07, Theorem 1.2] and that Corollary 3.5 can be applied. Hence, by Lemma 3.4 and (the remark in the proof of) Corollary 3.5, we may suppose that $f: X \rightarrow C$ itself is a good minimal model after possibly a finite base change and a birational modification along the special fiber.

We take a large and divisible integer $m$ such that $m K_{X / C}$ is Cartier and $\left|m K_{X / C}\right|$ is basepoint free. We take a general member $D \in\left|m K_{X / C}\right|$ and let $\pi: Y \rightarrow X$ be the cyclic cover associated with $D$ and $f_{Y}:=f \circ \pi: Y \rightarrow C$. By Lemma 3.7, the variety $Y$ has Gorenstein canonical singularities and $K_{Y}=\pi^{*}\left(m K_{X}\right)$. Let $Y_{0}$ be the special fiber of $f_{Y}$ over $0 \in C$, and 
let $\pi: Y_{0} \rightarrow X_{0}$ be the induced morphism. As $X_{0}$ has canonical singularities and $\left|m K_{X_{0}}\right|=$ $\left|m K_{X / C}\right|_{X_{0}} \mid$ is base-point free, by Lemma 3.7 again, $Y_{0}$ has Gorenstein canonical singularities and $K_{Y_{0}}=\pi^{*}\left(m K_{X_{0}}\right)$.

Recall that $f_{Y *} \mathcal{O}_{Y}\left(\ell K_{Y / C}\right)=\oplus_{i=1}^{m} f_{*} \mathcal{O}_{X}\left((m(\ell-1)+i) K_{X / C}\right)$ and $H^{0}\left(Y_{0}, \mathcal{O}_{Y_{0}}\left(\ell K_{Y_{0}}\right)\right)=$ $\oplus_{i=1}^{m} H^{0}\left(X_{0}, \mathcal{O}_{X_{0}}\left((m(\ell-1)+i) K_{X_{0}}\right)\right)$ for every integer $\ell>0$. We notice that every fiber near $Y_{0}$ (including $\left.Y_{0}\right)$ has canonical singularities and $f$ has the property $\left(P_{k}\right)$ for all $k>0$. This in particular implies that $f_{Y}$ has the property $\left(P_{\ell}\right)$ for all $\ell>0$. Now, $P_{1}\left(Y_{t}\right)=p_{g}\left(Y_{t}\right)>0$ for general $t \in C$. Then by [Wan03, Corollary 2.4], we see that $\omega_{1}\left(=g_{H}\right)$ for $Y \rightarrow C$ is incomplete at $0 \in C$. Then by the definition of $\omega_{m, D}$ for $X \rightarrow C$, the pseudo-metric $\omega_{m, D}$ is incomplete at $0 \in C$.

We add a supplement to [Wan03, Corollary 2.4]. We will not use it in this paper.

Lemma 3.11. Suppose in Setup 3.1 that $X_{0}$ is normal and has canonical singularities at worst and $p_{g}\left(X_{t}\right)>0$ for general $t \in C$. Then $0 \in C$ is at finite distance to a point $t \in C^{o}$ with respect to $g_{H}\left(=\omega_{1}\right)$; that is, $\omega_{1}$ is incomplete at $0 \in C$. Note that we do not suppose that $X_{0}$ is Gorenstein here.

Proof. We know $p_{g}\left(X_{0}\right)=p_{g}\left(X_{t}\right)$ for general $t \in C$, for example by [Tak07, Theorem 1.1]. If we take a semi-stable reduction $f^{\prime}: X^{\prime} \rightarrow C^{\prime}$ of $f$, by the invariant cycle theorem [Wan03, proof of Theorem 2.3], we have $p_{g}\left(X_{s}\right)=\sum_{i \in I} p_{g}\left(F_{i}^{\prime}\right)$, where $X_{0}^{\prime}=\sum_{i \in I} F_{i}^{\prime}$ is the decomposition into irreducible components of the special fiber of $f^{\prime}$. We are saying that $f^{\prime}: X^{\prime} \rightarrow C$ has the property $\left(P_{1}\right)$. Then by [Wan03, Theorem 2.3], we obtain our assertion.

\section{A filling-in problem, II}

We shall prove Theorem 1.4 in this section. Because of the nature of our problem in Theorem 1.4, we can actually suppose that $f: X \rightarrow C$ is semi-stable. We can take a finite morphism $\sigma: C^{\prime} \rightarrow C$ from a pointed smooth curve $\left(C^{\prime}, 0^{\prime}\right)$ with $\sigma\left(0^{\prime}\right)=0$ and a resolution of singularities $\alpha: \widetilde{X}^{\prime} \rightarrow$ $X^{\prime}:=X \times_{C} C^{\prime}$ of $X^{\prime}$ such that the induced family $f_{\widetilde{X}^{\prime}}: \widetilde{X}^{\prime} \rightarrow C^{\prime}$ becomes semi-stable around $0^{\prime} \in C^{\prime}$ and the special fiber $\tilde{X}_{0^{\prime}}^{\prime}$ is simple normal crossing. We shall consider $f_{\tilde{X}^{\prime}}: \tilde{X}^{\prime} \rightarrow C^{\prime}$ around $0^{\prime} \in C^{\prime}$ instead of $f: X \rightarrow C$ from the beginning. The following theorem is a precise version of Theorem 1.4.

Theorem 4.1. Suppose in Setup 3.1 that $X$ is smooth and that the special fiber $X_{0}=\sum_{i \in I} F_{i}$ of $f: X \rightarrow C$ over 0 is reduced and simple normal crossing. Then the following conditions are equivalent:

(1) For any good minimal model $f^{\prime}: X^{\prime} \rightarrow C$ of $X$ over $C$, the special fiber $X_{0}^{\prime}$ has only canonical singularities.

$\left(1^{\prime}\right)$ There exists a good minimal model $f^{\prime}: X^{\prime} \rightarrow C$ of $X$ over $C$ whose special fiber $X_{0}^{\prime}$ has only canonical singularities.

(2) For any integer $m$ that is large and divisible with respect to $f$ and any general $D \in$ $\left|m K_{X / C}\right|$, the pseudo-metric $\omega_{m, D}$ is incomplete at $0 \in C$. (See below for the terminology.)

$\left(2^{\prime}\right)$ There exists a good minimal model $f^{\prime}: X^{\prime} \rightarrow C$ of $X$ over $C$ such that for any integer $m>0$ with $m K_{X^{\prime}}$ Cartier and $\left|m K_{X^{\prime}}\right|$ base-point free, there exists a general member $D \in\left|m K_{X^{\prime} / C}\right|$ such that the pseudo-metric $\omega_{m, D}$ for $f^{\prime}: X^{\prime} \rightarrow C$ is incomplete at $0 \in C$. 


\section{S. TAKAYAMA}

(3) For any integer $m>0$, the morphism $f$ has the property $\left(P_{m}\right)$; namely, $\sum_{i \in I} P_{m}\left(F_{i}\right)=$ $P_{m}\left(X_{t}\right)$ holds for $t \in C^{o}$ general.

$\left(3^{\prime}\right)$ For any large and divisible integer $m$, the morphism $f$ has the property $\left(P_{m}\right)$.

Let us explain the terminology in statement (2) (in which the semi-stability of $f$ is irrelevant). We say that an integer $m>0$ is large and divisible with respect to $f$ if there exists a good minimal model $f^{\prime}: X^{\prime} \rightarrow C$ such that $m K_{X^{\prime}}$ is Cartier and $\left|m K_{X^{\prime}}\right|$ is base-point free. For such $m$, we say that $D \in\left|m K_{X / C}\right|$ is general if there exists a good minimal model $f^{\prime}: X^{\prime} \rightarrow C$ such that $m K_{X^{\prime}}$ is Cartier and $\left|m K_{X^{\prime}}\right|$ is base-point free and if there exists a general member $D^{\prime} \in\left|m K_{X^{\prime} / C}\right|$ which corresponds to $D$. The correspondence of $D$ and $D^{\prime}$ is as follows. As $X$ and $X^{\prime}$ are birational by a process of the minimal model program and both have canonical singularities, we have $H^{0}\left(X, m K_{X}\right) \cong H^{0}\left(X^{\prime}, m K_{X^{\prime}}\right)$ for every $m>0$, with isomorphism induced by the birational map between $X$ and $X^{\prime}$. Then $D$ is mapped to $D^{\prime}$. Hence, $D \in\left|m K_{X / C}\right|$ is general in the usual sense of algebraic geometry at least.

It is clear that the implications $(1) \Rightarrow\left(1^{\prime}\right),(2) \Rightarrow\left(2^{\prime}\right)$ and $(3) \Rightarrow\left(3^{\prime}\right)$ in Theorem 4.1 hold. We also have the implication $\left(3^{\prime}\right) \Rightarrow(1)$ by Theorem 1.1. If we suppose condition $\left(1^{\prime}\right)$, then $f^{\prime}: X^{\prime} \rightarrow C$ has the property $\left(P_{m}\right)$ for any $m>0$ by [Tak07, Theorem 1.2]. Then by point $(2)$ of Lemma 3.4, the minimal model $f$ has the property $\left(P_{m}\right)$ for any $m>0$; that is, condition (3) holds. Hence, conditions (1), (1'), (3), ( $\left.3^{\prime}\right)$ are equivalent. By [Wan03, Proposition 1.3], that is, Proposition 3.10, we have the implication $(1) \Rightarrow(2)$. Hence, it is enough to show that condition $\left(2^{\prime}\right)$ implies one of conditions $(1),\left(1^{\prime}\right),(3),\left(3^{\prime}\right)$. We shall show that condition $\left(2^{\prime}\right)$ implies condition $\left(3^{\prime}\right)$ by establishing the following alternative.

Proposition 4.2. Let $f: X \rightarrow C$ be as in Theorem 4.1. Let $m>0$ be a fixed integer and suppose that there exists a good minimal model $f^{\prime}: X^{\prime} \rightarrow C$ of $X$ over $C$ such that $m K_{X^{\prime}}$ is Cartier and $\left|m K_{X^{\prime}}\right|$ is base-point free. Then either $f$ has the property $\left(P_{m}\right)$, or $\omega_{m, D}$ for $f^{\prime}: X^{\prime} \rightarrow C$ is complete at $0 \in C$ for any $D \in\left|m K_{X^{\prime} / C}\right|$ which is $\left(X^{\prime}, X_{0}^{\prime}\right)$-general in the sense of Lemma 3.7 for the special fiber $X_{0}^{\prime} \subset X^{\prime}$ of $f^{\prime}$.

This proposition immediately shows the implication $\left(2^{\prime}\right) \Rightarrow\left(3^{\prime}\right)$ of Theorem 4.1.

Proof of the implication $\left(2^{\prime}\right) \Rightarrow\left(3^{\prime}\right)$ of Theorem 4.1. We suppose to the contrary that condition $\left(3^{\prime}\right)$ does not hold. We take any good minimal model $f^{\prime}: X^{\prime} \rightarrow C$ of $X$ over $C$ and any integers $M>0$ and $m_{0}>0$. Then we have to show that there exists an integer $m$ such that $m>M$, $m_{0} \mid m$ (that is, $m / m_{0}$ is an integer), $m K_{X^{\prime}}$ is Cartier and $\left|m K_{X^{\prime}}\right|$ is base-point free but $\omega_{m, D}$ is complete at $0 \in C$ for any $D \in\left|m K_{X^{\prime} / C}\right|$ which is $\left(X^{\prime}, X_{0}^{\prime}\right)$-general in the sense of Lemma 3.7 for the special fiber $X_{0}^{\prime} \subset X^{\prime}$ of $f^{\prime}$.

We take an integer $m^{\prime}>0$ such that $m^{\prime} K_{X^{\prime}}$ is Cartier and $\left|m^{\prime} K_{X^{\prime}}\right|$ is base-point free. As statement $\left(3^{\prime}\right)$ does not hold, there exists an integer $m$ such that $m>M$ and $\left(m^{\prime} m_{0}\right) \mid m$ but the property $\left(P_{m}\right)$ does not hold for $f$ and hence does not hold for $f^{\prime}$ by Lemma 3.4. For this $m$, it follows by Proposition 4.2 that $\omega_{m, D}$ is complete at $0 \in C$ for any $D \in\left|m K_{X^{\prime} / C}\right|$ which is $\left(X^{\prime}, X_{0}^{\prime}\right)$-general in the sense of Lemma 3.7.

The rest of this section is devoted to proving Proposition 4.2. We first recall necessary material for the proof. The property in Theorem $4.1(2)$ and $\left(2^{\prime}\right)$ is a property of the direct image $f_{*} K_{X / C}$ after a covering of $X$. A basic fact due to Griffiths [Gri70] and Fujita [Fuj78] is that the vector bundle $\left.\left(f_{*} K_{X / C}\right)\right|_{C^{o}}$ endowed with the fiberwise $L^{2}$-metric, say $h$, over $C^{o}$ has Griffiths semipositive curvature and $h$ extends as a singular Hermitian metric on $f_{*} K_{X / C}$ with Griffiths semipositive curvature. This is the formulation in [PT18], but Fujita already essentially observed 


\section{FILLING-IN PROBLEM AND DEGENERATIONS OF VARIETIES}

this. We first recall what is happening on $f_{*} K_{X / C}$ in the semi-stable case. We quote results of Yoshikawa in the simplest form which will be sufficient in our argument (and which might have been known before by Wang [Wan03, Theorem 2.1, Remark 2.2]). His argument relies on basic results in Hodge theory by Schmid [Sch73] and direct computations. We implicitly suppose that $f_{*} K_{X / C}$ is non-trivial.

Proposition 4.3 ([Yos10, Section 2.2]). Suppose in Setup 3.1 that $f: X \rightarrow C$ is semi-stable. Let $\left\{u_{1}, \ldots, u_{r}\right\}$ be a local frame of $f_{*} K_{X / C}$ at $0 \in C$, that is, $f_{*} K_{X / C}=\oplus_{i=1}^{r} \mathcal{O}_{C} u_{i}$ at $0 \in C$, and let $h(t)=\left(h_{i \bar{j}}(t)\right)$ on $C^{o}$, where $h_{i \bar{j}}=h\left(u_{i}, u_{j}\right)$, be the matrix representation of $h$ with respect to this basis. Then, there exist real analytic functions with $r \times r$ Hermitian matrices as values $A_{m}(t) \in C^{\omega}(C, \operatorname{Herm}(r))$ for $0 \leqslant m \leqslant n=\operatorname{dim} X-1$, with

$$
h(t)=\sum_{m=0}^{n} A_{m}(t)(-\log |t|)^{m} .
$$

Moreover, by defining the functions $a_{m}(t) \in C^{\omega}(C, \mathbb{R})$, for $0 \leqslant m \leqslant n r$, as

$$
\operatorname{det} h(t)=\sum_{m=0}^{n r} a_{m}(t)(-\log |t|)^{m},
$$

one has $a_{m}(0) \neq 0$ for some $0 \leqslant m \leqslant n r$.

We then introduce an invariant $\ell_{0}$ in the expression of $\operatorname{det} h(t)$ above:

$$
\ell_{0}=\max \left\{i ; 0 \leqslant i \leqslant n r, a_{i}(0) \neq 0\right\} .
$$

Proposition 4.4 ([Yos10, Theorem 1.1 or 4.1)]. Suppose in Setup 3.1 that $f: X \rightarrow C$ is semistable. The curvature form of the line bundle $\left.\operatorname{det}\left(f_{*} K_{X / C}\right)\right|_{C^{o}}$ has the following asymptotic behavior as $t \rightarrow 0$ :

$$
\left(\omega_{1}=\right) \frac{\sqrt{-1}}{2 \pi} \bar{\partial} \partial \log \operatorname{det} h(t)=\left(\frac{\ell_{0}}{|t|^{2}(-\log |t|)^{2}}+O\left(\frac{1}{|t|^{2}(-\log |t|)^{3}}\right)\right) \sqrt{-1} d t \wedge d \bar{t} .
$$

In the setting of Proposition 4.3, we have $\ell_{0}=0$ if and only if $\operatorname{det} h(t)$ is bounded as $t \rightarrow 0$. By Proposition 4.4 , in the case $\ell_{0}=0$, the pseudo-metric $\omega_{1}$ satisfies

$$
\omega_{1}=O\left(\frac{1}{|t|^{2}(-\log |t|)^{3}}\right) \sqrt{-1} d t \wedge d \bar{t},
$$

and therefore it is automatically incomplete. In the case $\ell_{0}>0$, the pseudo-metric $\omega_{1}$ is quasiisometric to $\left(|t|^{2}(-\log |t|)^{2}\right)^{-1} \sqrt{-1} d t \wedge d \bar{t}$, the so-called Poincaré growth, which is complete (see also [Wan03, Theorem 2.1, Remark 2.2]). Hence, in our problem, it is important to see the (un)boundedness of $\operatorname{det} h(t)$ as $t \rightarrow 0$. This is further reduced to an estimate of fiberwise $L^{2}$ norms of one section (without a semi-stability condition).

Lemma 4.5. Let $f: X \rightarrow C$ be as in Setup 3.1. Suppose that there exists a $u \in H^{0}\left(C, f_{*} K_{X / C}\right)$ such that $h(u, u)(t)$ is unbounded as $t \rightarrow 0$. Then det $h(t)$ is unbounded as $t \rightarrow 0$.

Proof. By Griffiths and Fujita, the fiberwise $L^{2}$-metric $h$ on $\left.\left(f_{*} K_{X / C}\right)\right|_{C^{o}}$ extends as a singular Hermitian metric on the vector bundle $f_{*} K_{X / C}$ with Griffiths semi-positive curvature. In our setting, $h$ is smooth on $C^{o}$ and may be singular at $0 \in C$ at most. Moreover, it can only have $0<\operatorname{det} h \leqslant+\infty$, because $\operatorname{det} h=e^{-\varphi}$ around $0 \in C$ for a plurisubharmonic (psh for short) function $\varphi$ on a neighborhood of $0 \in C$. 


\section{S. TAKAYAMA}

On the other hand, up to multiplying $u$ by a suitable power of $t^{-1}$, one may assume that $u$ is non-zero at $t=0$ (namely, $u$ does not vanish identically along $X_{0}$ viewed as an element of $\left.H^{0}\left(X, K_{X / C}\right)\right)$ and that $h(u, u)(t)$ is unbounded as $t \rightarrow 0$. Then, Lemma 4.5 follows directly from Lemma 4.6(3) below, which gives an abstract property of singular Hermitian vector bundles with semi-definite curvature.

We recall briefly the definition of singular Hermitian metrics (refer to [BP08, §3, p. 357] or [PT18, $\S 2])$. Let $E=B \times \mathbb{C}^{r}$ be a trivial vector bundle on a unit ball $B$ in $\mathbb{C}^{n}$. A singular Hermitian metric $h$ on $E$ is a measurable map $h: B \rightarrow \operatorname{Herm}(r)$ to the space of all $r \times r$ Hermitian matrices such that $0<\operatorname{det} h<+\infty$ almost everywhere on $B$. The metric $h$ is said to have Griffiths semi-negative curvature if $\log h(u, u)$ is psh on $U$ for any open set $U \subset B$ and any $u \in H^{0}(U, E)$. The metric $h$ is said to have Griffiths semi-positive curvature if the dual metric $h^{*}$ has Griffiths semi-negative curvature.

Lemma 4.6. Let $h=\left(h_{i \bar{j}}\right)$ be a singular Hermitian metric on a trivial vector bundle $E$ on a unit ball $B$ in $\mathbb{C}^{n}$ as above. Let $U \subset B$ be a smaller ball.

(1) Suppose that $h$ has Griffiths semi-negative curvature. Then there exists a constant $M>0$ such that $\left|h_{i j}\right| \leqslant M$ on $U$ for any entry. Let $0 \leqslant \lambda_{1}(x) \leqslant \lambda_{2}(x) \leqslant \cdots \leqslant \lambda_{r}(x)<+\infty$ be the eigenvalues of $h(x)$ at each point $x \in B$. Then we have $\lambda_{r}(x) \leqslant r M$ on $U$ and $\lambda_{1}(x) \geqslant(r M)^{1-r} \operatorname{det} h(x)$ on $U$.

(2) Suppose that $h$ has Griffiths semi-positive curvature. Then there exists a constant $M^{\prime}>0$ (independent of $\left.x \in U \backslash\{\operatorname{det} h=+\infty\}\right)$ such that $\lambda_{1}(x) \geqslant 1 /\left(r M^{\prime}\right)$ and $\lambda_{r}(x) \leqslant$ $\left(r M^{\prime}\right)^{r-1} \operatorname{det} h(x)$ on $U \backslash\{\operatorname{det} h=+\infty\}$, where $0 \leqslant \lambda_{1}(x) \leqslant \lambda_{2}(x) \leqslant \cdots \leqslant \lambda_{r}(x)<+\infty$ are the eigenvalues of $h(x)$ at $x \in B \backslash\{\operatorname{det} h=+\infty\}$. (In fact, $\lambda_{1}(x)>0$.)

(3) Suppose that $h$ has Griffiths semi-positive curvature. Suppose that there is a constant $A>0$ such that $\operatorname{det} h \leqslant A$ on $U \backslash\{\operatorname{det} h=+\infty\}$. Then there is a constant $A^{\prime}>0$ such that $\left|h_{i \bar{j}}\right| \leqslant$ $A^{\prime}$ on $U \backslash\{\operatorname{det} h=+\infty\}$ for any entry of $h$. In particular, if $u_{1}, \ldots, u_{r} \in H^{0}(B, E)$ are such that $E=\oplus_{i=1}^{r} \mathcal{O}_{B} u_{i}$ and if $h_{1 \overline{1}}=h\left(u_{1}, u_{1}\right)$ is unbounded from above on $U \backslash\{\operatorname{det} h=+\infty\}$, then det $h$ is unbounded from above on $U \backslash\{\operatorname{det} h=+\infty\}$.

Proof. (1) By definition, $\log h(u, u)$ is psh on $B$ for any $u \in H^{0}(B, E)$. In particular, $h(u, u)$ is non-negative and psh on $B$, and hence it is also locally bounded from above. Thus there exists a constant $M^{\prime}>0$ such that $h_{i \bar{i}} \leqslant M^{\prime}$ on $U$. As $h(x)$ is positive semi-definite, we have $\left|h_{i \bar{j}}(x)\right|^{2} \leqslant h_{i \bar{i}}(x) h_{j \bar{j}}(x)$ for every $i, j$. This proves the first assertion.

Given a matrix $P=\left(p_{i j}\right) \in M(r, \mathbb{C})$, we denote by $M:=\max \left\{\left|p_{i j}\right| ; 1 \leqslant i, j \leqslant r\right\}$ the maximum of the absolute value of its entries. Then we have $|\lambda| \leqslant r M$ for any eigenvalue of $P$. Note that $\operatorname{det} h=\lambda_{1} \lambda_{2} \cdots \lambda_{r} \leqslant \lambda_{1}(r M)^{r-1}$ at every $x \in U$.

(2) Take the dual and apply statement (1) as long as $\operatorname{det} h^{*} \neq 0$.

(3) We shall discuss this only on $U \backslash\{\operatorname{det} h=+\infty\}$. By statement (2) and the inequality $\operatorname{det} h \leqslant A$, we have $\operatorname{tr} h(x)=\lambda_{1}(x)+\cdots+\lambda_{r}(x) \leqslant r\left(r M^{\prime}\right)^{r-1} A$. As $h(x)$ is positive definite, we have $0<h_{i \bar{i}}(x)<\operatorname{tr} h(x)$ and $\left|h_{i \bar{j}}(x)\right|^{2} \leqslant h_{i \bar{i}}(x) h_{j \bar{j}}(x)$ for every $i, j$. We then obtain our bound.

We now go back to the discussion of Proposition 4.2. We suppose in Proposition 4.2 that $f$ does not have the property $\left(P_{m}\right)$. Then by Lemma 3.4 , the minimal model $f^{\prime}$ also does not have the property $\left(P_{m}\right)$. In particular, $X_{0}^{\prime}$ has a singularity worse than canonical thanks to the equivalence of statements $(1),\left(1^{\prime}\right),(3),\left(3^{\prime}\right)$ of Theorem 4.1. But still, $X_{0}^{\prime}$ has semi-log-canonical 


\section{FILLING-IN PROBLEM AND DEGENERATIONS OF VARIETIES}

singularities by Lemma 2.3(2). As $X_{0}^{\prime}$ is demi-normal [Kol13, Definition-Lemma 5.10], the scheme $X_{0}^{\prime}$ is normal if and only if $X_{0}^{\prime}$ is regular in codimension 1. Recall that the singularity type in codimension 1 of a semi-log-canonical variety is an ordinary node [Kol13, Definition 5.1]. So we can take a point $Q^{\prime} \in X_{0}^{\prime}$ such that

(i) $X_{0}^{\prime}$ is non-normal and $Q^{\prime}$ is a general point in Sing $X_{0}^{\prime}$ which is an ordinary node around there, or

(ii) $X_{0}^{\prime}$ is normal and $Q^{\prime}$ is a non-canonical singularity of $X_{0}^{\prime}$.

We note that the pair $\left(X^{\prime}, X_{0}^{\prime}\right)$ is lc by Lemma 3.9 as $X_{0}^{\prime}$ is semi-log-canonical. We take any general $D \in\left|m K_{X^{\prime} / C}\right|$ which is $\left(X^{\prime}, X_{0}^{\prime}\right)$-general in the sense of Lemma 3.7, and let $\pi: Y \rightarrow X^{\prime}$ be the $m$ th root of $D$ as in Lemma 3.7. We can suppose that $D$ does not contain $Q^{\prime}$ and can suppose that the induced family $f_{Y}:=f^{\prime} \circ \pi: Y \rightarrow C$ with $0 \in C$ satisfies Setup 3.1. Then we shall show the following.

Proposition 4.7. Suppose in Proposition 4.2 that $f$ does not have the property $\left(P_{m}\right)$. Let $D \in\left|m K_{X^{\prime} / C}\right|$ be a general member, $\pi: Y \rightarrow X^{\prime}$ the mth root of $D$ and $f_{Y}=f^{\prime} \circ \pi: Y \rightarrow C$ be the induced morphism (as above). Then there exist a section $u \in H^{0}\left(C, f_{Y *} K_{Y / C}\right.$ ), an open neighborhood $C_{1} \subset C$ of $0 \in C$ and a constant $A>0$ such that

$$
h(u, u)(t) \geqslant A(-\log |t|)
$$

holds for any $t \in C_{1} \backslash 0$.

Remark 4.8. The variety $Y$ in Proposition 4.7 has Gorenstein canonical singularities, $\left|K_{Y}\right|$ is base-point free, and the pair $\left(Y, Y_{0}\right)$ is lc by Lemma 3.7. As the map $\pi: Y \rightarrow X$ is unramified around over $Q^{\prime}$, the scheme $Y_{0}$ has the same property at every point of $\pi^{-1}\left(Q^{\prime}\right)$ as $X_{0}^{\prime}$ has at $Q^{\prime}$. In particular, we can take a point $Q \in Y_{0}$ such that

(i) $Y_{0}$ is non-normal and $Q$ is a general point in $\operatorname{Sing} Y_{0}$ which is an ordinary node around there, or

(ii) $Y_{0}$ is normal and $Q$ is a non-canonical singularity of $Y_{0}$.

Assuming Proposition 4.7 for the moment, we can prove Proposition 4.2 and hence Theorem 4.1, as shown below.

Proposition 4.9. In Proposition 4.7, the pseudo-metric $\omega_{1}$ for $f_{Y}: Y \rightarrow C$ (that is, $\omega_{m, D}$ for $\left.f^{\prime}: X^{\prime} \rightarrow C\right)$ is complete at $0 \in C$. In particular, Proposition 4.2 holds.

Proof of Proposition 4.9. We apply a semi-stable reduction theorem for $f_{Y}: Y \rightarrow C$. We take a finite morphism $\sigma: C^{\prime} \rightarrow C$ from a pointed smooth curve $\left(C^{\prime}, 0^{\prime}\right)$ with $\sigma^{-1}(0)=0^{\prime}$ and a resolution of singularities $\alpha: \widetilde{Y}^{\prime} \rightarrow Y^{\prime}:=Y \times_{C} C^{\prime}$ of $Y^{\prime}$ such that the induced family $f_{\widetilde{Y}^{\prime}}: \tilde{Y}^{\prime} \rightarrow C^{\prime}$ becomes semi-stable and the special fiber is simple normal crossing. We can suppose that $\alpha$ is biregular on $Y^{\prime} \backslash f_{Y^{\prime}}^{-1}\left(0^{\prime}\right)$. Let us consider the induced diagram

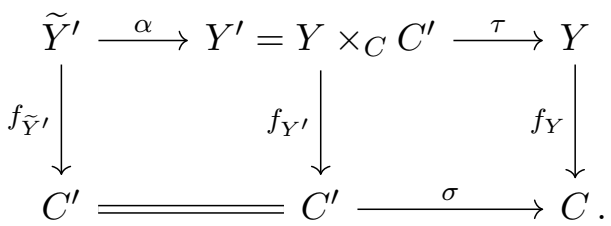

Note that $Y^{\prime}$ and $f_{Y^{\prime}}$ are smooth over $C^{\prime} \backslash 0^{\prime}$. We can further see that $Y^{\prime}$ is normal, $f_{Y^{\prime}}$ is lc (refer to [KM98, Definition 7.1]) and $K_{Y^{\prime} / C^{\prime}}=\tau^{*} K_{Y / C}$ (see the proof of [KM98, Lemma 7.6]). 


\section{S. TAKAYAMA}

In particular, $Y^{\prime}$ has canonical singularities at worst [KM98, Lemma 7.2], the divisor $K_{Y^{\prime}}$ is Cartier and $\left|K_{Y^{\prime}}\right|$ is base-point free. Moreover, $Y_{0^{\prime}}^{\prime}=Y_{0}$ by construction. Thus the properties of $f_{Y}: Y \rightarrow C$ mentioned in Remark 4.8 are preserved by a finite base change. Namely, $f_{Y^{\prime}}: Y^{\prime} \rightarrow C$ inherits the properties in Remark 4.8. We apply Proposition 4.7 for $f_{\widetilde{Y}^{\prime}}: \widetilde{Y}^{\prime} \rightarrow C^{\prime}$ and use Proposition 4.4 (thanks to Lemmas 4.5 and 4.6). We note that $\omega_{1}$ for $f_{Y^{\prime}}: Y^{\prime} \rightarrow C^{\prime}$ and $\omega_{1}$ for $f_{\widetilde{Y}^{\prime}}: \widetilde{Y}^{\prime} \rightarrow C^{\prime}$ are the same. Then we see that $\omega_{1}$ (say $\widetilde{\omega}_{1}^{\prime}$ ) for $f_{\widetilde{Y}^{\prime}}: \widetilde{Y}^{\prime} \rightarrow C^{\prime}$ is complete at $0^{\prime} \in C^{\prime}$. We also note that $\widetilde{\omega}_{1}^{\prime}=\sigma^{*} \omega_{1}$ on $C^{\prime} \backslash\left\{0^{\prime}\right\}$. Thus the completeness of $\omega_{1}$ at $0 \in C$ for $f_{Y}: Y \rightarrow C$ and the completeness of $\widetilde{\omega}_{1}^{\prime}$ at $0^{\prime} \in C^{\prime}$ for $f_{\widetilde{Y}^{\prime}}: \widetilde{Y}^{\prime} \rightarrow C^{\prime}$ are equivalent by the finite base change $\sigma$. Thus $\omega_{1}$ for $f_{Y}: Y \rightarrow C$ is complete at $0 \in C$.

Proof of Proposition 4.7. We shall write by $f: Y \rightarrow C$ instead of $f_{Y}$ and let $Y_{0}=\sum_{i \in I} F_{i}$. We note that $Y_{0}$ is reduced as $\pi$ is generically étale over $X_{0}$. We will not use $f: X \rightarrow C$ and $X_{0}=\sum_{i \in I} F_{i}$ in the proof. We have a special point $Q \in Y_{0}$.

Step 1. We first prepare to compute fiberwise integrals. We take a log-resolution $\alpha: \widetilde{Y} \rightarrow Y$ of the pair $\left(Y, Y_{0}\right)$ such that $\alpha$ is biholomorphic over $Y \backslash Y_{0}$. Let $\widetilde{F}=\sum_{i \in I} \widetilde{F}_{i}$ be the strict transform of $Y_{0}$, which is a disjoint union of smooth divisors. We set $\tilde{f}=f \circ \alpha: \widetilde{Y} \rightarrow C$ and let $\widetilde{Y}_{0}=\widetilde{f}^{*}(t=0)=\widetilde{F}+\sum_{j \in J} p_{j} E_{j}$, where the $E_{j}$ are $\alpha$-exceptional prime divisors and the $p_{j}>0$ are integers. We also have $K_{\tilde{Y} / Y}=K_{\tilde{Y}}-\alpha^{*} K_{Y}=\sum_{j \in J} k_{j} E_{j}$ with non-negative integers $k_{j} \geqslant 0$. Here we recall that $Y$ has Gorenstein canonical singularities. We let $k_{i}=0(i \in I)$ and $\widetilde{F}=\sum_{i \in I} p_{i} \widetilde{F}_{i}$ with $p_{i}=1$ formally.

Step 1.1. We take a small open neighborhood $U$ of $Q \in Y$ and a local frame $\eta \in H_{\widetilde{Y}}^{0}\left(U, K_{Y}\right)$. We also take a point $\widetilde{Q} \in \alpha^{-1}(Q) \subset \widetilde{Y}$ and local coordinates $w=\left(w_{1}, \ldots, w_{n}, w_{n+1}\right)$ in $\widetilde{Y}$ centered at $\widetilde{Q}$, which are defined on an open subset containing $W=\left\{w \in \mathbb{C}^{n+1} ;\left|w_{i}\right| \leqslant 1\right.$ for any $1 \leqslant i \leqslant$ $n+1\}$, which satisfies $\alpha(W) \subset U$. The point $\widetilde{Q}$ will be specified later. We can assume without loss of generalities that, on $W$,

$$
\begin{aligned}
t & =\widetilde{f}(w)=w_{1}^{p_{1}} w_{2}^{p_{2}} \cdots w_{n+1}^{p_{n+1}}, \\
\alpha^{*} \eta & =(\operatorname{Jac} \alpha) d w_{1} \wedge \cdots \wedge d w_{n+1}=w_{1}^{k_{1}} \cdots w_{n+1}^{k_{n+1}} d w_{1} \wedge \cdots \wedge d w_{n+1},
\end{aligned}
$$

where the $p_{j}$ and $k_{j}$ are non-negative integers as we indicated above.

As the pair $\left(Y, Y_{0}\right)$ is lc, if we write $K_{\widetilde{Y}}+\widetilde{F}=\alpha^{*}\left(K_{Y}+Y_{0}\right)+E$ with an $\alpha$-exceptional $E=\sum_{j \in J} e_{j} E_{j}$, then $e_{j} \geqslant-1$ for all $j$. In particular, if $E_{j}=\left\{w_{j}=0\right\}$, then $e_{j}=k_{j}-p_{j} \geqslant-1$. We rewrite this formula as $K_{\widetilde{Y}}+D=\alpha^{*}\left(K_{Y}+Y_{0}\right)$ with $D=\sum d_{\ell} D_{\ell}:=\widetilde{F}-E$. If $d_{\ell}<0$, then $D_{\ell}$ is one of the $E_{j}$ and hence $D_{\ell}$ is $\alpha$-exceptional. In this sense, $\alpha_{*} D$ defines an effective divisor. It is clear that $-\left(K_{\widetilde{Y}}+D\right)=-\alpha^{*}\left(K_{Y}+Y_{0}\right)$ is $\alpha$-nef and $\alpha$-big as $\alpha$ is birational. Then by the connectedness lemma [KM98, Theorem 5.48], the divisor $\sum_{i ; d_{i} \geqslant 1} D_{i}=\widetilde{F}+\sum_{e_{j}=-1} E_{j}$ is connected in a neighborhood of $\alpha^{-1}(Q)$.

(i) Suppose that $Y_{0}$ is non-normal and $Q$ is a general point of $\operatorname{Sing} Y_{0}$. Then $\left.\alpha\right|_{\widetilde{F}}: \widetilde{F} \rightarrow Y_{0}$ is finite morphism around $Q$, and in fact $\left(\left.\alpha\right|_{\widetilde{F}}\right)^{-1}(Q)$ consists of two different points and is, in particular, disconnected. By the connectedness lemma above, $\sum_{i ; d_{i} \geqslant 1} D_{i}=\widetilde{F}+\sum_{e_{j}=-1} E_{j}$ is connected in a neighborhood of $\alpha^{-1}(Q)$. Thus there exist irreducible components $\widetilde{F}_{i}$ and $E_{j}$ such that $\widetilde{F}_{i} \cap E_{j} \cap \alpha^{-1}(Q) \neq \emptyset$.

(ii) Suppose that $Y_{0}$ is normal and $Q$ is a non-canonical singularity of $Y_{0}$. Then $\widetilde{F}$ is a smooth model of $Y_{0}$, and $K_{\widetilde{F}}=\alpha^{*} K_{Y_{0}}+\left.E\right|_{\widetilde{F}}$ by adjunction. As $Y_{0}$ is not canonical at $Q$, there exists an 


\section{Filling-IN PROBLEM AND DEGENERATIONS OF VARIETIES}

irreducible component $E_{j}$ such that $\widetilde{F} \cap E_{j} \cap \alpha^{-1}(Q) \neq \emptyset$ and $e_{j}<0$, that is,$e_{j}=-1$. This is a connectedness lemma in principle, though the connectedness lemma is not used directly.

In both cases (i) and (ii), we can take irreducible components $\widetilde{F}_{i}$ and $E_{j}$ with $e_{j}=-1$ such that $\widetilde{F}_{i} \cap E_{j} \cap \alpha^{-1}(Q) \neq \emptyset$. We now specify a point $\widetilde{Q} \in \alpha^{-1}(Q)$ by requiring $\widetilde{Q} \in \widetilde{F}_{i} \cap E_{j} \cap \alpha^{-1}(Q)$. We then retake our local coordinate $w=\left(w_{1}, \ldots, w_{n}, w_{n+1}\right)$ on $W \subset \widetilde{Y}$ such that $\widetilde{F}_{i}=\left\{w_{n+1}=\right.$ $0\}$ and $E_{j}=\left\{w_{n}=0\right\}$. In particular, $\widetilde{Q} \in\left\{w_{n}=w_{n+1}=0\right\}, p_{n+1}=1$ and $p_{n}>0$.

As $\tilde{f}$ is flat, $\tilde{f}(W)$ contains an open neighborhood of $t=0$. We take a point $t \in \tilde{f}(W) \backslash 0$. Then the smooth fiber $\widetilde{Y}_{t} \cap W$ can be regarded as the graph of a function

$$
w_{n+1}=G\left(w_{1}, \ldots, w_{n}\right)=\frac{t}{w_{1}^{p_{1}} \cdots w_{n}^{p_{n}}}
$$

of $\left(w_{1}, \ldots, w_{n}\right)$. We set $W_{t}^{*}=\left\{\left(w_{1}, \ldots, w_{n}\right) \in \mathbb{C}^{n} ;\left|w_{j}\right| \leqslant 1\right.$ if $p_{j}=0,2^{-1 /(n-1) p_{j}} \leqslant\left|w_{j}\right| \leqslant 1$ if $p_{j} \neq 0$ and $\left.1 \leqslant j \leqslant n-1,|2 t|^{1 / p_{n}} \leqslant\left|w_{n}\right| \leqslant 1\right\}$. We note that $\left(w_{1}, \ldots, w_{n}, G\left(w_{1}, \ldots, w_{n}\right)\right) \in$ $\tilde{Y}_{t} \cap W$ if $\left(w_{1}, \ldots, w_{n}\right) \in W_{t}^{*}$. In fact, we see that $\left|t /\left(w_{1}^{p_{1}} \cdots w_{n}^{p_{n}}\right)\right| \leqslant 1$ for $\left(w_{1}, \ldots, w_{n}\right) \in W_{t}^{*}$.

Step 1.2. By the relation $\tilde{f}^{*} d t / \widetilde{f}(w)=\sum_{j=1}^{n+1} p_{j}\left(d w_{j} / w_{j}\right)$, we have the equality $d w_{n+1}=$ $\left(w_{n+1} / \widetilde{f}(w)\right) \tilde{f}^{*} d t$ modulo $d w_{1}, \ldots, d w_{n}$. Then we can write

$$
\begin{aligned}
\alpha^{*} \eta & =(\operatorname{Jac} \alpha) d w_{1} \wedge \cdots \wedge d w_{n+1} \\
& =\frac{w_{n+1}}{\widetilde{f}(w)}(\operatorname{Jac} \alpha) d w_{1} \wedge \cdots \wedge d w_{n} \wedge \widetilde{f}^{*} d t .
\end{aligned}
$$

To be more precise, if we let

$$
\sigma=\frac{w_{n+1}}{\widetilde{f}(w)}(\operatorname{Jac} \alpha) d w_{1} \wedge \cdots \wedge d w_{n}
$$

then $\sigma$ is holomorphic on $W \backslash \tilde{Y}_{0}$ and $\left.\left(\alpha^{*} \eta\right)\right|_{W \backslash \widetilde{Y}_{0}}=\sigma \wedge \widetilde{f}^{*} d t$ holds. We note that

$$
\frac{w_{n+1}}{\widetilde{f}(w)}(\operatorname{Jac} \alpha)=\prod_{j=1}^{n} w_{j}^{k_{j}-p_{j}}=w_{1}^{k_{1}-p_{1}} \cdots w_{n-1}^{k_{n-1}-p_{n-1}} w_{n}^{-1} .
$$

Then we have

$$
\begin{aligned}
\int_{\widetilde{Y}_{t} \cap W}(-1)^{n^{2} / 2} \sigma \wedge \bar{\sigma} & =\int_{\widetilde{Y}_{t} \cap W} \prod_{j=1}^{n}\left|w_{j}\right|^{2\left(k_{j}-p_{j}\right)} \bigwedge_{j=1}^{n} \sqrt{-1} d w_{j} \wedge d \overline{w_{j}} \\
& \geqslant \int_{W_{t}^{*}} \prod_{j=1}^{n}\left|w_{j}\right|^{2\left(k_{j}-p_{j}\right)} \bigwedge_{j=1}^{n} \sqrt{-1} d w_{j} \wedge d \overline{w_{j}} .
\end{aligned}
$$

The last integral is estimated from below by a product of

$$
\begin{aligned}
& \prod_{1 \leqslant j \leqslant n-1, p_{j}=0} \int_{\left|w_{j}\right| \leqslant 1}\left|w_{j}\right|^{2\left(k_{j}-p_{j}\right)} \sqrt{-1} d w_{j} \wedge d \overline{w_{j}},
\end{aligned}
$$

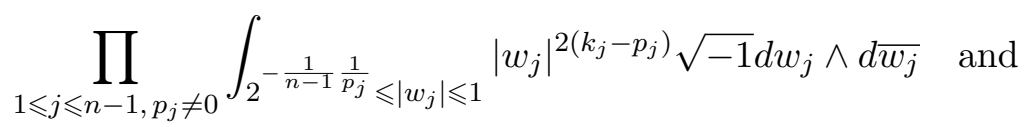

$$
\begin{aligned}
& \int_{|2 t|^{1 / p_{n} \leqslant\left|w_{n}\right| \leqslant 1}}\left|w_{n}\right|^{-2} \sqrt{-1} d w_{n} \wedge d \overline{w_{n}} .
\end{aligned}
$$

The former two integrals are estimated from below by a constant $A^{\prime}>0$ depending only on $k_{j}$, 


\section{S. TAKAYAMA}

$p_{j}, n$. The third integral is

$$
\iint_{|2 t|^{1 / p_{n} \leqslant r \leqslant 1,0 \leqslant \theta \leqslant 2 \pi}} \frac{2 r d r d \theta}{r^{2}}=4 \pi\left(-\log |2 t|^{1 / p_{n}}\right) .
$$

Thus there exist a neighborhood $C_{1}$ of $0 \in C$ and a constant $A>0$ independent of $t \in C_{1} \backslash 0$ such that

$$
\int_{\widetilde{Y}_{t} \cap W}(-1)^{n^{2} / 2} \sigma \wedge \bar{\sigma} \geqslant A(-\log |t|)
$$

holds for any $t \in C_{1} \backslash 0$.

Step 2. We now complete the proof of Proposition 4.7. As $\left|K_{Y}\right|$ is base-point free, we can take a section $\eta \in H^{0}\left(Y, K_{Y}\right)$ which gives a local frame of $K_{Y}$ around $Q$. By a choice of a trivialization $d t \in H^{0}\left(C, K_{C}\right)$ of $K_{C}$, we identify $H^{0}\left(Y, K_{Y / C}\right) \cong H^{0}\left(Y, K_{Y}\right)$. We take $u \in H^{0}\left(Y, K_{Y / C}\right)$ which corresponds to $\eta \in H^{0}\left(Y, K_{Y}\right)$ via this identification. Then $u(d t)=\eta \in H^{0}\left(Y, K_{Y}\right)$. Here we regard $H^{0}\left(C, f_{*} K_{Y / C}\right)$ as $\operatorname{Hom}\left(K_{C}, f_{*} K_{Y}\right)$, and hence regard $u$ as a homomorphism $u: K_{C} \rightarrow f_{*} K_{Y}$ and $u(d t) \in H^{0}\left(C, f_{*} K_{Y}\right)=H^{0}\left(Y, K_{Y}\right)$. Then

$$
h(u, u)(t) \geqslant \int_{\widetilde{Y}_{t} \cap W}(-1)^{n^{2} / 2} \sigma \wedge \bar{\sigma} \geqslant A(-\log |t|)
$$

holds as in the statement.

\section{ACKNOWLEDGEMEnTs}

The author would like to thank the referee for his/her careful reading and many suggestions and comments.

\section{REFERENCES}

AK00 D. Abramovich and K. Karu, Weak semistable reduction in characteristic 0, Invent. Math. 139 (2000), no. 2, 241-273; doi:10.1007/s002229900024.

BP08 B. Berndtsson and M. Păun, Bergman kernels and the pseudoeffectivity of relative canonical bundles, Duke Math. J. 145 (2008), no. 2, 341-378; doi:10.1215/00127094-2008-054.

DS14 S. Donaldson and S. Sun, Gromov-Hausdorff limits of Kähler manifolds and algebraic geometry, Acta Math. 213 (2014), no. 1, 63-106; doi:10.1007/s11511-014-0116-3.

EV92 H. Esnault and E. Viehweg, Lectures on vanishing theorems, DMV Seminar, vol. 20 (Birkhäuser Verlag, Basel, 1992); doi:10.1007/978-3-0348-8600-0.

Fuj11 O. Fujino, Semi-stable minimal model program for varieties with trivial canonical divisor, Proc. Japan Acad. Ser. A Math. Sci. 87 (2011), no. 3, 25-30; doi:10.3792/pjaa.87.25.

Fuj16a _ Direct images of relative pluricanonical bundles, Algebr. Geom. 3 (2016), no. 1, 50-62; doi:10.14231/AG-2016-003.

Fuj16b_, Corrigendum to "Direct images of relative pluricanonical bundles" [Algebr. Geom. 3 (2016), no. 1, 50-62], Algebr. Geom. 3 (2016), no. 2, 261-263; doi:10.14231/AG-2016-012.

Fuj78 T. Fujita, On Kähler fiber spaces over curves, J. Math. Soc. Japan 30 (1978), no. 4, 779-794; doi:10.2969/jmsj/03040779.

Gri70 P. A. Griffiths, Periods of integrals on algebraic manifolds. III. Some global differential-geometric properties of the period mapping, Publ. Math. Inst. Hautes Études Sci. (1970), no. 38, 125-180; doi:10.1007/BF02684654. 


\section{FILLING-IN PROBLEM AND DEGENERATIONS OF VARIETIES}

Har77 R. Hartshorne, Algebraic geometry, Grad. Texts in Math., vol. 52 (Springer-Verlag, New York Heidelberg, 1977); doi:10.1007/978-1-4757-3849-0.

HM07 C. D. Hacon and J. McKernan, On Shokurov's rational connectedness conjecture, Duke Math. J. 138 (2007), no. 1, 119-136; doi:10.1215/S0012-7094-07-13813-4.

HX13 C. D. Hacon and C. Xu, Existence of log canonical closures, Invent. Math. 192 (2013), no. 1, 161-195; doi:10.1007/s00222-012-0409-0.

Ish14 S. Ishii, Introduction to singularities (Springer, Tokyo, 2014); doi:10.1007/978-4-431-55081-5.

Kar00 K. Karu, Minimal models and boundedness of stable varieties, J. Algebraic Geom. 9 (2000), no. 1, 93-109.

Kaw91 Y. Kawamata, On the length of an extremal rational curve, Invent. Math. 105 (1991), no. 3, 609-611; doi:10.1007/BF01232281.

Kaw99_, Deformations of canonical singularities, J. Amer. Math. Soc. 12 (1999), no. 1, 85-92; doi:10.1090/S0894-0347-99-00285-4.

KM98 J. Kollár and S. Mori, Birational geometry of algebraic varieties, Cambridge Tracts in Math., vol. 134 (Cambridge Univ. Press, Cambridge, 1998); doi:10.1017/CB09780511662560.

Kol13 J. Kollár, Singularities of the minimal model program, Cambridge Tracts in Math., vol. 200 (Cambridge Univ. Press, Cambridge, 2013); doi:10.1017/CB09781139547895.

Nak04 N. Nakayama, Zariski-decomposition and abundance, MSJ Memoirs, vol. 14 (Math. Soc. Japan, Tokyo, 2004); doi:10.2969/msjmemoirs/014010000.

PT18 M. Păun and S. Takayama, Positivity of twisted relative pluricanonical bundles and their direct images, J. Algebraic Geom. 27 (2018), no. 2, 211-272; doi:10.1090/jag/702.

Pău07 M. Păun, Siu's invariance of plurigenera: a one-tower proof, J. Differential Geom. 76 (2007), no. 3, 485-493; doi:10.4310/jdg/1180135695.

Sch73 W. Schmid, Variation of Hodge structure: the singularities of the period mapping, Invent. Math. 22 (1973), 211-319; doi:10.1007/BF01389674.

Siu98 Y.-T. Siu, Invariance of plurigenera, Invent. Math. 134 (1998), no. 3, 661-673; doi:10.1007/s002220050276.

Siu02 _ Extension of twisted pluricanonical sections with plurisubharmonic weight and invariance of semipositively twisted plurigenera for manifolds not necessarily of general type, Complex Geometry (Göttingen, 2000) (Springer, Berlin, 2002), 223-277; doi:10.1007/978-3-642-56202-0_15.

Tak07 S. Takayama, On the invariance and the lower semi-continuity of plurigenera of algebraic varieties, J. Algebraic Geom. 16 (2007), no. 1, 1-18; doi:10.1090/S1056-3911-06-00455-3.

Tak08_, On uniruled degenerations of algebraic varieties with trivial canonical divisor, Math. Z. 259 (2008), no. 3, 487-501; doi:10.1007/s00209-007-0235-z.

Tak15_ On moderate degenerations of polarized Ricci-flat Kähler manifolds, J. Math. Sci. Univ. Tokyo 22 (2015), no. 1, 469-489; http://www.ms.u-tokyo.ac.jp/journal/pdf/jms220115. pdf.

Tos15 V. Tosatti, Families of Calabi-Yau manifolds and canonical singularities, Int. Math. Res. Not. 2015 (2015), no. 20, 10586-10594; doi:10.1093/imrn/rnv001.

Wan03 C.-L. Wang, Quasi-Hodge metrics and canonical singularities, Math. Res. Lett. textbf10 (2003), no. 1, 57-70; doi:10.4310/MRL.2003.v10.n1.a7.

Yos10 K.-I. Yoshikawa, On the boundary behavior of the curvature of $L^{2}$-metrics, 2010, arXiv: 1007.2836.

Zha16 Y. Zhang, Completion of the moduli space for polarized Calabi-Yau manifolds, J. Differential Geom. 103 (2016), no. 3, 521-544; doi:10.4310/jdg/1468517503.

Shigeharu Takayama taka@ms.u-tokyo.ac.jp

Graduate School of Mathematical Sciences, The University of Tokyo, 3-8-1 Komaba, Meguro-ku, Tokyo, 153-8914, Japan 